\title{
An energy stable coupling procedure for the compressible and incompressible Navier-Stokes equations
}

Fatemeh Ghasemi and J an Nordström

The self-archived postprint version of this journal article is available at Linköping University Institutional Repository (DiVA):

http:// urn.kb.se/ resolve?urn=urn:nbn:se:liu:diva-159116

N.B.: When citing this work, cite the original publication.

Ghasemi, F., Nordström, J ., (2019), An energy stable coupling procedure for the compressible and incompressible Navier-Stokes equations, J ournal of Computational Physics, 396, 280-302.

https:// doi.org/ 10.1016/j.jcp.2019.07.022

Original publication available at:

https:// doi.org/ 10.1016/j.jcp.2019.07.022

Copyright:

Elsevier http:/ / www.elsevier.com/

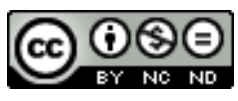




\title{
An Energy Stable Coupling Procedure for the Compressible and Incompressible Navier-Stokes Equations
}

\author{
Fatemeh Ghasemi \& Jan Nordström \\ Computational Mathematics, Department of Mathematics, Linköping University, \\ SE-581 83 Linköping, Sweden \\ fatemeh.ghasemi@liu.se, jan.nordstrom@liu.se
}

\begin{abstract}
The coupling of the compressible and incompressible Navier-Stokes equations is considered. Our ambition is to take a first step towards a provably well posed and stable coupling procedure. We study a simplified setting with a stationary planar interface and small disturbances from a steady background flow with zero velocity normal to the interface.

The simplified setting motivates the use of the linearized equations, and we derive interface conditions such that the continuous problem satisfy an energy estimate. The interface conditions can be imposed both strongly and weakly. It is shown that the weak and strong interface imposition produce similar continuous energy estimates.

We discretize the problem in time and space by employing finite difference operators that satisfy a summation-by-parts rule. The interface and initial conditions are imposed weakly using a penalty formulation. It is shown that the results obtained for the weak interface conditions in the continuous case, lead directly to stability of the fully discrete problem.
\end{abstract}

Keywords: Compressible fluid, incompressible fluid, Navier-Stokes equations, energy estimate, interface conditions, stability

\section{Introduction}

The ocean-atmosphere coupling is one of the major factors influencing weather and climate. In particular it is critical for predicting changes in global temperature patterns and warming trends $[1,2,3,4]$. This multiphase 
coupled problem can be handled with three different methodologies: one can treat both phases as compressible, both phases as incompressible or the gas as compressible and the liquid as incompressible.

The fully compressible fluid model is limited to liquids where acceptable compressibility models for their evolution [5] exist. The fully incompressible model is valid as long as the density variations in the gas phase can be neglected [6]. These two models lead to a conventional coupling procedure, since the same set of equations govern both phases. The compressibleincompressible coupling is in many cases more realistic and general, but it is not straightforward, since the set of governing equations differ $[7,8]$.

Another challenge apart from compressibility of the fluids, is posed by the presence of viscosity. In $[7,8]$, the authors considered inviscid fluids modeled by the Euler equations. In this paper, we consider the compressibleincompressible approach for viscous fluids modeled by the Navier-Stokes equations and to a large extent follow the general procedure used in $[9,10]$. Our ambition is to present a first step towards a provably well posed and stable coupling procedure. This rigorous mathematical treatment is more straightforward by employing the approximations specified in the beginning of Section 2 below. In particular, we will show that the continuous analysis of the interface treatment, lead directly to stability for summation-by-parts (SBP) based schemes.

As our numerical method, we will use finite differences but the analysis is valid also for genuinely multi-dimensional SBP schemes [11, 12] as well as finite volume [13, 14], flux reconstruction schemes $[15,16,17]$ and discontinuous Galerkin schemes $[18,19]$ on SBP form. Our ambition is to derive a provably accurate and stable coupling procedure for modeling the interaction between compressible and incompressible fluids. To make the analysis transparent and easy to follow, the linearized problem with a stationary interface and steady background flow with zero velocity normal to the interface will be used.

The rest of the paper proceeds as follows. In Section 2, we introduce the equations and derive the number and form of the interface conditions. Section 3 deals with obtaining a bound for the energy rate for both strong and weak imposition of interface conditions. In Section 4, we present the semidiscrete formulation and derive stability conditions. Numerical experiments are performed in Section 5 to show the accuracy of the procedure. Finally, we summarize and draw conclusions in Section 6 . 


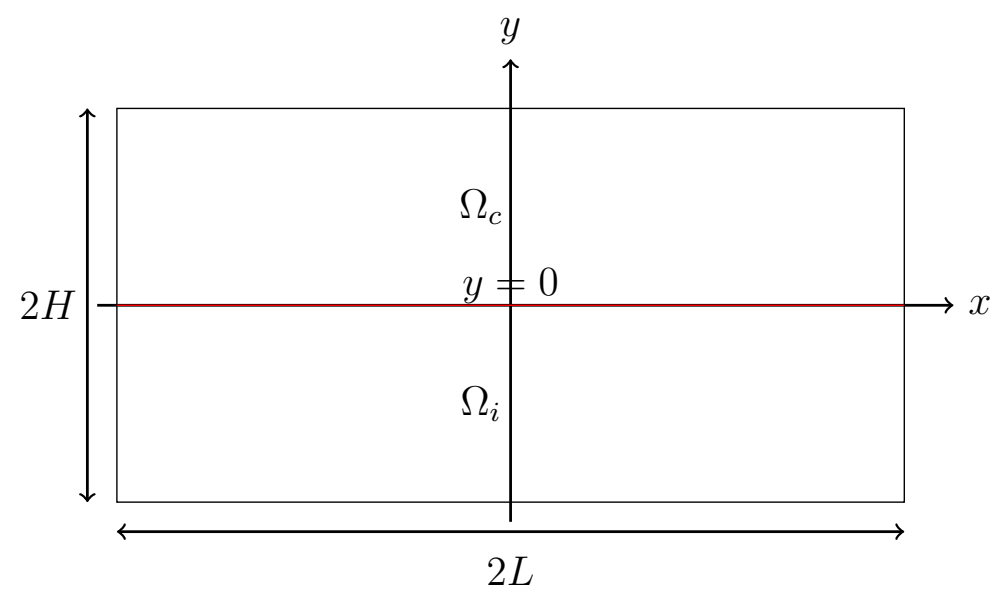

Figure 1: A schematic of the domains and interface $y=0$.

\section{The continuous problem}

With the coupling of ocean-atmosphere in mind, we consider a two dimensional setting where the two solutions vary slightly around a constant background state which is continuous across the interface and has zero normal velocity. Based on this assumption and the additional approximation that we consider a fixed interface at $y=0$, we study the linearized problems. The compressible gas occupies $\left.\Omega_{c}=\right]-L,+L[\times] 0,+H[$ and the incompressible fluid $\left.\Omega_{i}=\right]-L,+L[\times]-H, 0[$. We further assume that the linearized solutions have compact support in $]-L,+L[\times]-H,+H[$ and focus on the conditions along the interface $y=0$, see Figure 1 .

Remark 1. As stated above, we have for ease of presentation and analysis assumed that the interface position is fixed in time. For a read up on moving interface, see [20, 21].

\subsection{The compressible Navier-Stokes equations}

The linearized and symmetrized compressible Navier-Stokes equations $[22,23]$ are

$$
U_{t}+A_{1} U_{x}+A_{2} U_{y}=\epsilon\left(F_{x}^{c}+G_{y}^{c}\right)
$$

where $U=[\bar{c} \rho / \sqrt{\gamma}, \bar{\rho} u, \bar{\rho} v, \bar{\rho} T / \bar{c} \sqrt{\gamma(\gamma-1)}]^{T}$ and $(\rho, u, v, T)$ is the perturbation. Throughout this paper, we use subscripts to denote partial derivatives, 
i.e. $U_{t}=\partial U / \partial t$ in (1). The viscous fluxes are given by

$$
F^{c}=A_{11} U_{x}+A_{12} U_{y}, \quad G^{c}=A_{21} U_{x}+A_{22} U_{y},
$$

and

$$
\begin{aligned}
& A_{1}=\left[\begin{array}{cccc}
\bar{u} & \bar{c} & 0 & 0 \\
\bar{c} & \overline{\sqrt{\gamma}} & & \bar{c} \sqrt{\frac{\gamma-1}{\gamma}} \\
\overline{\sqrt{\gamma}} & \bar{u} & 0 & 0 \\
0 & 0 & \bar{u} \\
0 & \bar{c} \sqrt{\frac{\gamma-1}{\gamma}} & 0 & \bar{u}
\end{array}\right], A_{11}=\frac{1}{\bar{\rho}}\left[\begin{array}{cccc}
0 & 0 & 0 & 0 \\
0 & \bar{\lambda}+2 \bar{\mu} & 0 & 0 \\
0 & 0 & \bar{\mu} & 0 \\
0 & 0 & 0 & \frac{\gamma \bar{\kappa}}{\operatorname{Pr}}
\end{array}\right],
\end{aligned}
$$

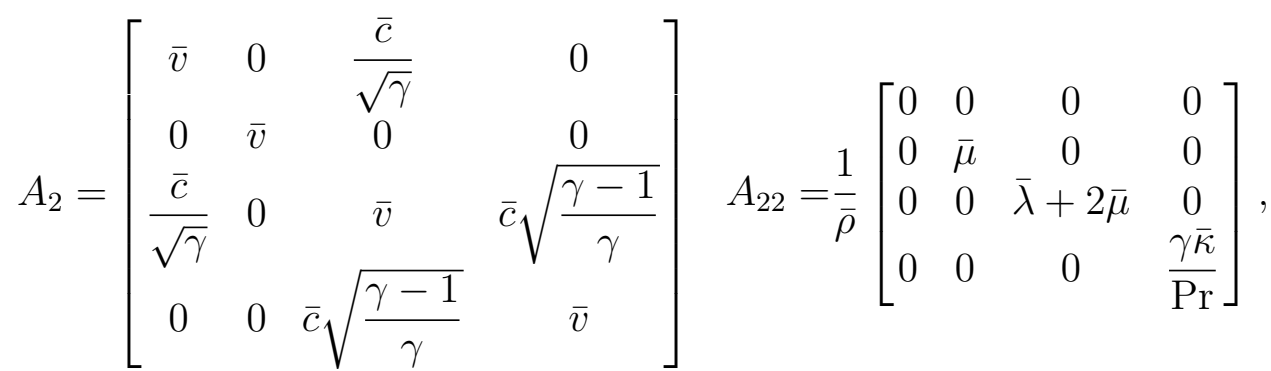

$$
\begin{aligned}
& A_{12}=A_{21}^{T}=\frac{1}{\bar{\rho}}\left[\begin{array}{cccc}
0 & 0 & 0 & 0 \\
0 & 0 & \bar{\lambda} & 0 \\
0 & \bar{\mu} & 0 & 0 \\
0 & 0 & 0 & 0
\end{array}\right] \text {. }
\end{aligned}
$$

In (3), the overbar denotes the constant state around which we have linearized. The dependent variables are the density $\rho$, the velocities $u, v$ in the $x$ and $y$ direction, respectively and the temperature $T$. We assume an ideal fluid and hence the equation of state is $\gamma p=\rho T$, where $p$ is the pressure and $\gamma=c_{p} / c_{v}$ is the ratio of the specific heats. Furthermore, we have used the speed of sound $\bar{c}$, the shear and second viscosity $\bar{\mu}, \bar{\lambda}$, the thermal conductivity $\bar{\kappa}$, the Prandtl number $\operatorname{Pr}=\mu_{\infty} c_{p} / \kappa_{\infty}$ and $\epsilon=1 / R e$ where $R e=\rho_{\infty} U_{\infty} L / \mu_{\infty}$ is the Reynolds number. The infinity subscript denotes the reference value and $L$ is a characteristic length scale. For simplicity and ease of presentation, in the remainder of the paper, we assume that $\bar{v}=0$. 


\subsection{Incompressible Navier-Stokes equations}

The linearized incompressible Navier-Stokes equations are

$$
\begin{aligned}
\hat{\rho}\left(\tilde{u}_{x}+\tilde{v}_{y}\right) & =0, \\
\hat{\rho}\left(\tilde{u}_{t}+\hat{u} \tilde{u}_{x}+\hat{v} \tilde{u}_{y}\right) & =-\tilde{p}_{x}+\epsilon \hat{\mu}\left(\tilde{u}_{x x}+\tilde{u}_{y y}\right), \\
\hat{\rho}\left(\tilde{v}_{t}+\hat{u} \tilde{v}_{x}+\hat{v} \tilde{v}_{y}\right) & =-\tilde{p}_{y}+\epsilon \hat{\mu}\left(\tilde{v}_{x x}+\tilde{v}_{y y}\right) .
\end{aligned}
$$

The dependent variables $\tilde{u}, \tilde{v}$ and $\tilde{p}$, represent the perturbation around the constant states of the velocity field $(\hat{u}, \hat{v})$ and the pressure. The parameters $\hat{\mu}, \hat{\rho}$ denote the constant viscosity and constant density.

Remark 2. We have multiplied the first equation in (4) with $\hat{\rho}$ to get a similar form of the continuity equation as in the compressible equations (1).

The fact that $\tilde{u}_{x}+\tilde{v}_{y}=0$ implies that $\left(\tilde{u}_{x}+\tilde{v}_{y}\right)_{x}=\left(\tilde{u}_{x}+\tilde{v}_{y}\right)_{y}=0$, and the system (4) can be rewritten as

$$
\tilde{I}_{3} V_{t}+B_{1} V_{x}+B_{2} V_{y}=\epsilon\left(F_{x}^{i}+G_{y}^{i}\right),
$$

where the viscous fluxes are

$$
F^{i}=B_{11} V_{x}+B_{12} V_{y}, \quad G^{i}=B_{21} V_{x}+B_{22} V_{y} .
$$

In (5) and (6), $\tilde{I}_{3}=\operatorname{diag}(0,1,1), V=[\tilde{p}, \hat{\rho} \tilde{u}, \hat{\rho} \tilde{v}]^{T}$ and

$$
\begin{array}{rlrl}
B_{1} & =\left[\begin{array}{lll}
0 & 1 & 0 \\
1 & \hat{u} & 0 \\
0 & 0 & \hat{u}
\end{array}\right], & B_{11}=\frac{1}{\hat{\rho}}\left[\begin{array}{ccc}
0 & 0 & 0 \\
0 & 2 \hat{\mu} & 0 \\
0 & 0 & \hat{\mu}
\end{array}\right], \\
B_{2}=\left[\begin{array}{lll}
0 & 0 & 1 \\
0 & \hat{v} & 0 \\
1 & 0 & \hat{v}
\end{array}\right], & B_{22}=\frac{1}{\hat{\rho}}\left[\begin{array}{ccc}
0 & 0 & 0 \\
0 & \hat{\mu} & 0 \\
0 & 0 & 2 \hat{\mu}
\end{array}\right], \\
B_{12}=B_{21}^{T}=\frac{1}{\hat{\rho}}\left[\begin{array}{lll}
0 & 0 & 0 \\
0 & 0 & 0 \\
0 & \hat{\mu} & 0
\end{array}\right] . &
\end{array}
$$

Also in this case, we assume $\hat{v}=0$ in the following.

Remark 3. By transforming equation (4) into (5) using the gradients of the divergence relation, the structure in (6) becomes similar to the one in (2). Later, we will show that this transformation simplifies the proof of the well-posedness.

Remark 4. The parameter $\epsilon$ is the same for the both the compressible and incompressible equations, since we have used the same reference values. 


\subsection{Interface conditions}

The number of boundary/interface conditions can be derived using the energy method $[23,10]$ or the Laplace transform technique $[24,25]$. In this paper, we will use the energy method as our main analysis tool and derive an equation for the energy-rate. For clarity, we ignore the outer boundary conditions, which have been studied in [23, 26, 27, 28, 29,30,31], and focus only on the interface at $y=0$.

\subsubsection{The number of interface conditions}

The energy method (multiplying (1) and (5) by the transpose of the solution and integrating over the spatial domains), together with the use of the Green-Gauss theorem, yields

$$
\frac{d}{d t}\left(\|U\|_{2}^{2}+\|V\|_{\tilde{I}_{3}}^{2}\right)+2 \epsilon D I S_{1}+2 \epsilon D I S_{2}=-\left.\int_{-L}^{+L} W^{T} E W\right|_{y=0} d x,
$$

where $\|U\|_{2}=\int_{\Omega_{c}} U^{T} U d \Omega,\|V\|_{\tilde{I}_{3}}=\int_{\Omega_{i}} V^{T} \tilde{I}_{3} V d \Omega$. In (7), $W=\left[U, \epsilon G^{c}, \epsilon G^{i}, V\right]^{T}$ and

$$
E=\left[\begin{array}{cccc}
-A_{2} & \tilde{I}_{4} & 0 & 0 \\
\tilde{I}_{4} & 0 & 0 & 0 \\
0 & 0 & 0 & -\tilde{I}_{3} \\
0 & 0 & -\tilde{I}_{3} & B_{2}
\end{array}\right], \quad \tilde{I}_{4}=\operatorname{diag}(0,1,1,1) .
$$

The dissipative terms in (7) are given by

$$
D I S_{1}=\int_{\Omega_{c}}\left[\begin{array}{c}
U_{x} \\
U_{y}
\end{array}\right]^{T} D_{1}\left[\begin{array}{c}
U_{x} \\
U_{y}
\end{array}\right] d \Omega, D I S_{2}=\int_{\Omega_{i}}\left[\begin{array}{c}
V_{x} \\
V_{y}
\end{array}\right]^{T} D_{2}\left[\begin{array}{c}
V_{x} \\
V_{y}
\end{array}\right] d \Omega,
$$

where

$$
D_{1}=\left[\begin{array}{ll}
A_{11} & A_{12} \\
A_{21} & A_{22}
\end{array}\right], \quad D_{2}=\left[\begin{array}{ll}
B_{11} & B_{12} \\
B_{21} & B_{22}
\end{array}\right]
$$

are positive semi-definite [27, 28, 31, 32].

Remark 5. The first element of the vectors $G^{c}$ and $G^{i}$ is zero. The corresponding rows and columns in $E$ are therefore empty.

The negative terms in the quadratic form $W^{T} E W$ may cause growth and must be bounded. This means that the number of interface conditions is 
compressible fluid

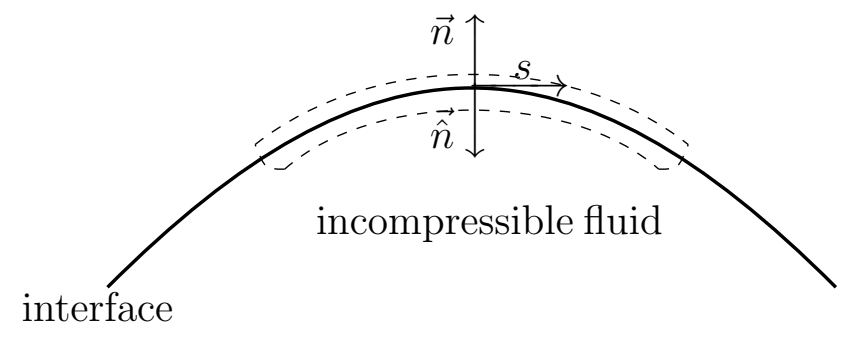

Figure 2: A sketch of a fluid-fluid interface with a thin control volume.

equal to the number of negative eigenvalues of the matrix $E[10,23]$. The matrix $E$ has five negative eigenvalues

$\Lambda^{-}=\left\{-1,-1,-\sqrt{2},-\sqrt{1+\eta_{1}},-\sqrt{1+\eta_{2}}\right\}$, where $\eta_{1,2}=\frac{\bar{c}^{2}}{2} \pm \sqrt{\frac{\bar{c}^{4}}{4}+\frac{\bar{c}^{2}(\gamma-1)}{\gamma},}$

four zero eigenvalues and five positive eigenvalues where $\Lambda^{+}=-\Lambda^{-}$. The five negative eigenvalues implies that five interface conditions are required.

Remark 6. Since the matrix $E$ is real and symmetric, all eigenvalues are real. It can be shown that $1+\eta_{1,2}>0$.

\subsubsection{The form of the interface conditions}

The following interface conditions were derived in [20,33, 34, 35, 36], where an infinitely thin control volume that follows the interface was considered (see Figure 2). Conservation of momentum in the control volume demands that

$$
\sigma \vec{n}=-\tilde{\sigma} \overrightarrow{\hat{n}},
$$

where $\overrightarrow{\hat{n}}=-\vec{n}$ is the unit normal vector shown in Figure 2. In (9), $\sigma=$ $p I_{2}-\epsilon \tau, \tilde{\sigma}=\tilde{p} I_{2}-\epsilon \tilde{\tau}$ and

$$
\begin{aligned}
\tau & =\left[\begin{array}{cc}
2 \bar{\mu} u_{x}+\bar{\lambda}\left(u_{x}+v_{y}\right) & \bar{\mu}\left(u_{y}+v_{x}\right) \\
\bar{\mu}\left(u_{y}+v_{x}\right) & 2 \bar{\mu} v_{y}+\bar{\lambda}\left(u_{x}+v_{y}\right)
\end{array}\right], \\
\tilde{\tau} & =\left[\begin{array}{cc}
2 \hat{\mu} \tilde{u}_{x} & \hat{\mu}\left(\tilde{u}_{y}+\tilde{v}_{x}\right) \\
\hat{\mu}\left(\tilde{u}_{y}+\tilde{v}_{x}\right) & 2 \hat{\mu} \tilde{v}_{y}
\end{array}\right],
\end{aligned}
$$

are the viscous stress tensors in each fluid. Multiplying (9) with $\vec{n}^{T}$ yields

$$
p-\epsilon \vec{n}^{T} \tau \vec{n}=\tilde{p}-\epsilon \vec{n}^{T} \tilde{\tau} \vec{n},
$$


which prescribes continuity on the normal stress across the interface. Multiplying (9) with the unit tangential vector $\vec{s}^{T}$, yields

$$
\epsilon \vec{S}^{T} \tau \vec{n}=-\epsilon \vec{S}^{T} \tilde{\tau} \overrightarrow{\hat{n}}
$$

which prescribes continuity of shear stresses across the interface.

Continuity of velocity across the interface leads to

$$
\vec{u}=\overrightarrow{\tilde{u}}
$$

where $\vec{u}=(u, v)^{T}, \overrightarrow{\tilde{u}}=(\tilde{u}, \tilde{v})^{T}$. The vector condition (12) consists of two parts. The first one is the continuity of the tangential velocities,

$$
\vec{u} \cdot \vec{s}=\overrightarrow{\tilde{u}} \cdot \vec{s}
$$

The second one is the kinematic condition which is the continuity of the normal velocities

$$
\vec{u} \cdot \vec{n}=-\overrightarrow{\tilde{u}} \cdot \overrightarrow{\hat{n}}
$$

Continuity of the normal component stems from the no flow condition through the interface, whereas the tangential components are continuous because both fluids are viscous [34].

In our setup at the interface $y=0$, we have $\vec{n}=[0,1]^{T}, \overrightarrow{\hat{n}}=[0,-1]^{T}$ and $\vec{s}=[1,0]^{T}$ and consequently, the interface conditions (10), (11), (13) and (14) become

$$
\phi=\tilde{\phi}, \quad \phi=\left[\begin{array}{c}
u \\
p-2 \epsilon \bar{\mu} v_{y}-\epsilon \bar{\lambda}\left(u_{x}+v_{y}\right) \\
v \\
\epsilon \bar{\mu}\left(u_{y}+v_{x}\right)
\end{array}\right], \quad \tilde{\phi}=\left[\begin{array}{c}
\tilde{u} \\
\tilde{p}-2 \epsilon \hat{\mu} \tilde{v}_{y} \\
\tilde{v} \\
\epsilon \hat{\mu}\left(\tilde{u}_{y}+\tilde{v}_{x}\right)
\end{array}\right],
$$

which are only four interface conditions.

\section{The energy estimate}

In this section, we show that the four interface conditions given in (15) do not suffice for well-posedness. We start by strongly imposing the interface conditions. 


\subsection{Strongly imposed interface conditions}

We derive the energy rate in the semi-norm

$$
\|U\|_{2}^{2}+\delta_{1}\|V\|_{\tilde{I}_{3}}^{2}=\int_{\Omega_{c}} U^{T} U d \Omega+\delta_{1} \int_{\Omega_{i}} V^{T} \tilde{I}_{3} V d \Omega
$$

The unknown weight $\delta_{1}>0$ will be determined below [37]. Applying the energy method to (1) and (5) leads to

$$
\frac{d}{d t}\left(\|U\|_{2}^{2}+\delta_{1}\|V\|_{\tilde{I}_{3}}^{2}\right)+2 \epsilon D I S_{1}+2 \epsilon \delta_{1} D I S_{2}=I T F
$$

where

$$
I T F=\left.\int_{-L}^{+L}\left(U^{T}\left(A_{2} U-2 \epsilon G^{c}\right)-\delta_{1} V^{T}\left(B_{2} V-2 \epsilon G^{i}\right)\right)\right|_{y=0} d x
$$

With straightforward algebraic manipulations, one can show that the specific choice

$$
\delta_{1}=\frac{\bar{\rho}}{\hat{\rho}}
$$

leads to

$$
U^{T}\left(A_{2} U-2 \epsilon G^{c}\right)=-\phi^{T} \mathcal{A} \phi-2 \bar{\kappa} \beta T T_{y}
$$

and

$$
\delta_{1} V^{T}\left(B_{2} V-2 \epsilon G^{i}\right)=-\tilde{\phi}^{T} \mathcal{A} \tilde{\phi}
$$

where

$$
\mathcal{A}=\left[\begin{array}{cccc}
0 & 0 & 0 & \bar{\rho} \\
0 & 0 & -\bar{\rho} & 0 \\
0 & -\bar{\rho} & 0 & 0 \\
\bar{\rho} & 0 & 0 & 0
\end{array}\right], \quad \beta=\frac{\epsilon \bar{\rho}}{\operatorname{Pr} \bar{c}^{2}(\gamma-1)}
$$

Remark 7. The diagonal elements in the matrices $A_{2}$ and $B_{2}$ are zeros since $\bar{v}=\hat{v}=0$. This means that there are no quadratic $\phi$ terms in (18) and (19).

Remark 8. Relation (19) holds due to the transformation of (4) to (5).

Remark 9. The weight $\delta_{1}>0$ is determined in (17) such that a bound is obtained. 
By using (18) and (19), the interface term (16) can be rewritten as

$$
I T F=-\left.\int_{-L}^{+L}\left(\phi^{T} \mathcal{A} \phi-\tilde{\phi}^{T} \mathcal{A} \tilde{\phi}+2 \bar{\kappa} \beta T T_{y}\right)\right|_{y=0} d x .
$$

Inserting the interface conditions $\phi=\tilde{\phi}$ into (20) yields

$$
\frac{d}{d t}\left(\|U\|_{2}^{2}+\delta_{1}\|V\|_{\tilde{I}_{3}}^{2}\right)+2 \epsilon D I S_{1}+2 \epsilon \delta_{1} D I S_{2}=-\left.2 \bar{\kappa} \beta \int_{-L}^{+L} T T_{y}\right|_{y=0} d x
$$

which is indefinite and prevents us from getting an estimate. However, as mentioned above, we have only imposed four interface conditions, not five. Hence, we must add an additional condition.

\subsection{Added relations for heat distribution}

As our added relations, we consider the heat distribution in an incompressible fluid described by

$$
\hat{\rho}\left(\tilde{T}_{t}+\hat{u} \tilde{T}_{x}+\hat{v} \tilde{T}_{y}\right)=\frac{\epsilon \hat{\kappa}}{\hat{\operatorname{Pr}}}\left(\tilde{T}_{x x}+\tilde{T}_{y y}\right)
$$

where the term related to the dissipation of mechanical energy has been ignored. In (21), $\hat{\operatorname{Pr}}=\mu_{\infty} \tilde{c}_{p} / \kappa_{\infty}$ is the Prandtl number, $\tilde{T}$ is the temperature and $\hat{\kappa}$ is the thermal conductivity. Note that (21) is not required in order to solve (4). One can first solve (4) for the velocity and pressure distribution without knowing the temperature. Next, (21) can be solved to find the temperature distribution.

By adding the heat equation (21) to (5), we get the new system of equations

$$
\tilde{I}_{4} V_{t}+B_{1} V_{x}+B_{2} V_{y}=\epsilon\left(F_{x}^{i}+G_{y}^{i}\right),
$$

where the viscous fluxes are

$$
F^{i}=B_{11} V_{x}+B_{12} V_{y}, \quad G^{i}=B_{21} V_{x}+B_{22} V_{y}
$$


In $(22)$ and $(23), V=[\tilde{p}, \hat{\rho} \tilde{u}, \hat{\rho} \tilde{v}, \hat{\rho} \tilde{T}]^{T}$ and

$$
\begin{aligned}
& B_{1}=\left[\begin{array}{llll}
0 & 1 & 0 & 0 \\
1 & \hat{u} & 0 & 0 \\
0 & 0 & \hat{u} & 0 \\
0 & 0 & 0 & \hat{u}
\end{array}\right], \\
& B_{2}=\left[\begin{array}{llll}
0 & 0 & 1 & 0 \\
0 & \hat{v} & 0 & 0 \\
1 & 0 & \hat{v} & 0 \\
0 & 0 & 0 & \hat{v}
\end{array}\right], \\
& B_{12}=
\end{aligned}
$$

Remark 10. By applying the same technique as in Section 2.3.1, one can show that the coupled systems (1) and (22) require six interface conditions.

We will provide and study two different coupling relations for the temperature.

3.2.1. Continuity of temperature and heat flux across the interface

Continuity of temperature and heat flux require [38]

$$
T=\tilde{T}, \quad \bar{\kappa} \nabla T \cdot \vec{n}=-\hat{\kappa} \nabla \tilde{T} \cdot \overrightarrow{\hat{n}}
$$

where $\vec{n}=[0,1]^{T}$ and $\overrightarrow{\hat{n}}=[0,-1]^{T}$ at the interface $y=0$. Now, we update the interface conditions (15) and rewrite as

$$
\phi_{1}=\tilde{\phi}_{1}, \quad \phi_{1}=\left[\begin{array}{c}
u \\
p-2 \epsilon \bar{\mu} v_{y}-\epsilon \bar{\lambda}\left(u_{x}+v_{y}\right) \\
v \\
\epsilon \bar{\mu}\left(u_{y}+v_{x}\right) \\
T \\
\bar{\kappa} T_{y}
\end{array}\right], \quad \tilde{\phi}_{1}=\left[\begin{array}{c}
\tilde{u} \\
\tilde{p}-2 \epsilon \hat{\mu} \tilde{v}_{y} \\
\tilde{v} \\
\epsilon \hat{\mu}\left(\tilde{u}_{y}+\tilde{v}_{x}\right) \\
\tilde{T} \\
\hat{\kappa} \tilde{T}_{y}
\end{array}\right]
$$


Remark 11. The vectors $\phi_{1}$ and $\tilde{\phi}_{1}$ in (25) written in operator form are

$$
\begin{aligned}
& \phi_{1}=H U, \quad H=H_{0}+H_{x} \frac{\partial}{\partial x}+H_{y} \frac{\partial}{\partial y} \\
& \tilde{\phi}_{1}=\tilde{H} V, \quad \tilde{H}=\tilde{H}_{0}+\tilde{H}_{x} \frac{\partial}{\partial x}+\tilde{H}_{y} \frac{\partial}{\partial y}
\end{aligned}
$$

where

$$
\begin{aligned}
& H_{0}=\left[\begin{array}{cccc}
0 & \frac{1}{\bar{\rho}} & 0 & 0 \\
\frac{\bar{c}}{\sqrt{\gamma}} & 0 & 0 & \bar{c} \sqrt{\frac{\gamma-1}{\gamma}} \\
0 & 0 & \frac{1}{\bar{\rho}} & 0 \\
0 & 0 & 0 & 0 \\
0 & 0 & 0 & \frac{\bar{c} \sqrt{\gamma(\gamma-1)}}{\bar{\rho}} \\
0 & 0 & 0 & 0
\end{array}\right], \quad H_{x}=\left[\begin{array}{cccc}
0 & 0 & 0 & 0 \\
0 & -\frac{\epsilon \bar{\lambda}}{\bar{\rho}} & 0 & 0 \\
0 & 0 & 0 & 0 \\
0 & 0 & \frac{\epsilon \bar{\mu}}{\bar{\rho}} & 0 \\
0 & 0 & 0 & 0 \\
0 & 0 & 0 & 0
\end{array}\right] \\
& H_{y}=\left[\begin{array}{cccc}
0 & 0 & 0 & 0 \\
0 & 0 & -\frac{\epsilon(2 \bar{\mu}+\bar{\lambda})}{\bar{\rho}} & 0 \\
0 & 0 & 0 & 0 \\
0 & \frac{\epsilon \bar{\mu}}{\bar{\rho}} & 0 & 0 \\
0 & 0 & 0 & 0 \\
0 & 0 & 0 & \bar{\kappa} \frac{\bar{c} \sqrt{\gamma(\gamma-1)}}{\bar{\rho}}
\end{array}\right], \quad \tilde{H}_{0}=\left[\begin{array}{cccc}
0 & \frac{1}{\hat{\rho}} & 0 & 0 \\
1 & 0 & 0 & 0 \\
0 & 0 & \frac{1}{\hat{\rho}} & 0 \\
0 & 0 & 0 & 0 \\
0 & 0 & 0 & \frac{1}{\hat{\rho}} \\
0 & 0 & 0 & 0
\end{array}\right], \\
& \tilde{H}_{x}=\left[\begin{array}{cccc}
0 & 0 & 0 & 0 \\
0 & 0 & 0 & 0 \\
0 & 0 & 0 & 0 \\
0 & 0 & \frac{\epsilon \hat{\mu}}{\hat{\rho}} & 0 \\
0 & 0 & 0 & 0 \\
0 & 0 & 0 & 0
\end{array}\right], \quad \tilde{H}_{y}=\left[\begin{array}{cccc}
0 & 0 & 0 & 0 \\
0 & 0 & -\frac{2 \epsilon \hat{\mu}}{\hat{\rho}} & 0 \\
0 & 0 & 0 & 0 \\
0 & \frac{\epsilon \hat{\mu}}{\hat{\rho}} & 0 & 0 \\
0 & 0 & 0 & 0 \\
0 & 0 & 0 & \frac{\hat{\kappa}}{\hat{\rho}}
\end{array}\right] .
\end{aligned}
$$

The operators $H$ and $\tilde{H}$ will be used in the weak interface procedure below. 


\subsubsection{A convective interface condition}

In the presence of convective heating (or cooling) at an ocean-atmosphere surface, the interface condition can be written as [16, 39, 40]

$$
\bar{\kappa} \nabla T \cdot \vec{n}=\alpha(T-\tilde{T}), \quad \bar{\kappa} \nabla T \cdot \vec{n}=-\hat{\kappa} \nabla \tilde{T} \cdot \overrightarrow{\hat{n}}
$$

where $\alpha>0$ is the convection heat transfer coefficient.

The interface conditions (15) and (28) can be formulated as

$$
\phi_{2}=\tilde{\phi}_{2}, \quad \phi_{2}=\left[\begin{array}{c}
u \\
p-2 \epsilon \bar{\mu} v_{y}-\epsilon \bar{\lambda}\left(u_{x}+v_{y}\right) \\
v \\
\epsilon \bar{\mu}\left(u_{y}+v_{x}\right) \\
\bar{\kappa} T_{y}-\alpha T \\
\alpha T
\end{array}\right], \quad \tilde{\phi}_{2}=\left[\begin{array}{c}
\tilde{u} \\
\tilde{p}-2 \epsilon \hat{\mu} \tilde{v}_{y} \\
\tilde{v} \\
\epsilon \hat{\mu}\left(\tilde{u}_{y}+\tilde{v}_{x}\right) \\
-\alpha \tilde{T} \\
\hat{\kappa} \tilde{T}_{y}+\alpha \tilde{T}
\end{array}\right]
$$

Remark 12. The vectors $\phi_{2}$ and $\tilde{\phi}_{2}$ in (29) written in operator form are

$$
\begin{aligned}
& \phi_{2}=H U, \quad H=H_{0}+H_{x} \frac{\partial}{\partial x}+H_{y} \frac{\partial}{\partial y} \\
& \tilde{\phi}_{2}=\tilde{H} V, \quad \tilde{H}=\tilde{H}_{0}+\tilde{H}_{x} \frac{\partial}{\partial x}+\tilde{H}_{y} \frac{\partial}{\partial y}
\end{aligned}
$$

where the operators $H_{x}, \tilde{H}_{x}$ and $\tilde{H}_{y}$ are the same as in (27) while

$$
H_{0}=\left[\begin{array}{cccc}
0 & \overline{\bar{\rho}} & 0 & 0 \\
\bar{c} & 0 & 0 & \bar{c} \sqrt{\frac{\gamma-1}{\gamma}} \\
\frac{\sqrt{\gamma}}{\gamma} & 0 & \frac{1}{\bar{\rho}} & 0 \\
0 & 0 & 0 & 0 \\
0 & 0 & 0 & -\alpha \frac{\bar{c} \sqrt{\gamma(\gamma-1)}}{\bar{\rho}} \\
0 & 0 & 0 & \alpha \frac{\bar{c} \sqrt{\gamma(\gamma-1)}}{\bar{\rho}}
\end{array}\right], \quad \tilde{H}_{0}=\left[\begin{array}{cccc}
0 & \frac{1}{\hat{\rho}} & 0 & 0 \\
1 & 0 & 0 & 0 \\
0 & 0 & \frac{1}{\hat{\rho}} & 0 \\
0 & 0 & 0 & 0 \\
0 & 0 & 0 & -\frac{\alpha}{\hat{\rho}} \\
0 & 0 & 0 & \frac{\alpha}{\hat{\rho}}
\end{array}\right],
$$




$$
H_{y}=\left[\begin{array}{cccc}
0 & 0 & 0 & 0 \\
0 & 0 & -\frac{\epsilon(2 \bar{\mu}+\bar{\lambda})}{\bar{\rho}} & 0 \\
0 & 0 & 0 & 0 \\
0 & \frac{\epsilon \bar{\mu}}{\bar{\rho}} & 0 & 0 \\
0 & 0 & 0 & \bar{\kappa} \frac{\bar{c} \sqrt{\gamma(\gamma-1)}}{\bar{\rho}} \\
0 & 0 & 0 & 0
\end{array}\right] .
$$

The operators $H$ and $\tilde{H}$ will be used in the weak interface procedure below.

\subsubsection{The strong energy estimate}

We can now prove

Proposition 1. The coupled problem (1) and (22) with the interface conditions (25) or (29) satisfy an energy estimate.

Proof. As in Section 3.1, the energy rate will be derived in a semi-norm

$$
\|U\|_{2}^{2}+\|V\|_{\mathcal{H} \tilde{I}_{4}}^{2}=\int_{\Omega_{c}} U^{T} U d \Omega+\int_{\Omega_{i}} V^{T} \mathcal{H} \tilde{I}_{4} V d \Omega, \quad \mathcal{H}=\delta_{1} \tilde{\mathcal{H}},
$$

where $\tilde{\mathcal{H}}=\operatorname{diag}\left(1,1,1, \delta_{2}\right)$. The weight $\delta_{1}$ is given in (17). We will later determine $\delta_{2}>0$ such that $\mathcal{H}$ in (31) becomes a norm [37]. Applying the energy method to the new coupled systems (1) and (22) leads to

$$
\frac{d}{d t}\left(\|U\|_{2}^{2}+\|V\|_{\mathcal{H} \tilde{I}_{4}}^{2}\right)+2 \epsilon D I S_{1}+2 \epsilon \widetilde{D I S}_{2}=I T F
$$

where

$$
I T F=\left.\int_{-L}^{+L}\left(U^{T}\left(A_{2} U-2 \epsilon G^{c}\right)-V^{T} \mathcal{H}\left(B_{2} V-2 \epsilon G^{i}\right)\right)\right|_{y=0} d x
$$

Using the weighted norm will change the dissipation term related to the incompressible equations to

$$
\widetilde{D I S}_{2}=\int_{\Omega_{i}}\left[\begin{array}{c}
V_{x} \\
V_{y}
\end{array}\right]^{T} \tilde{D}_{2}\left[\begin{array}{c}
V_{x} \\
V_{y}
\end{array}\right] d \Omega
$$


where

$$
\tilde{D}_{2}=\delta_{1}\left[\begin{array}{cc}
\tilde{\mathcal{H}} B_{11} & \tilde{\mathcal{H}} B_{12} \\
\tilde{\mathcal{H}} B_{21} & \tilde{\mathcal{H}} B_{22}
\end{array}\right]
$$

Since $B_{12}=B_{21}^{T}$, as given in (24), we have

$$
\tilde{D}_{2}^{T}=\delta_{1}\left[\begin{array}{ll}
B_{11} \tilde{\mathcal{H}} & B_{21}^{T} \tilde{\mathcal{H}} \\
B_{12}^{T} \tilde{\mathcal{H}} & B_{22} \tilde{\mathcal{H}}
\end{array}\right]=\delta_{1}\left[\begin{array}{cc}
B_{11} \tilde{\mathcal{H}} & B_{12} \tilde{\mathcal{H}} \\
B_{21} \tilde{\mathcal{H}} & B_{22} \tilde{\mathcal{H}}
\end{array}\right]
$$

Furthermore, the matrices $B_{11}, B_{22}$ and $\tilde{\mathcal{H}}$ are diagonal which leads to $B_{11} \tilde{\mathcal{H}}=$ $\tilde{\mathcal{H}} B_{11}$ and $B_{22} \tilde{\mathcal{H}}=\tilde{\mathcal{H}} B_{22}$. The last row of the matrices $B_{12}$ and $B_{21}$ is zero, which implies that $B_{12} \tilde{\mathcal{H}}=B_{12}=\tilde{\mathcal{H}} B_{12}$ and $B_{21} \tilde{\mathcal{H}}=B_{21}=\tilde{\mathcal{H}} B_{21}$. This leads to

$$
\tilde{D}_{2}^{T}=\delta_{1}\left[\begin{array}{cc}
\tilde{\mathcal{H}} B_{11} & \tilde{\mathcal{H}} B_{12} \\
\tilde{\mathcal{H}} B_{21} & \tilde{\mathcal{H}} B_{22}
\end{array}\right]=\tilde{D}_{2} .
$$

The matrix $\tilde{D}_{2}$ is symmetric and a product of two positive semi-definite matrices, which implies that $\widetilde{D I S}_{2} \geq 0$ (see Theorem 7.5 of [41]).

The first term in ITF in (33) is the same as the one in (18) and the second term can be written as

$$
V^{T} \mathcal{H}\left(B_{2} V-2 \epsilon G^{i}\right)=-\tilde{\phi}^{T} \mathcal{A} \tilde{\phi}+2 \epsilon \delta_{2} \frac{\hat{\kappa} \hat{\rho} \tilde{T} \tilde{T}_{y}}{\hat{\operatorname{Pr}}}
$$

which is similar to (19) with one more term due to the added heat equation (21). The specific choice

$$
\delta_{2}=\left(\frac{\bar{\rho}}{\hat{\rho}}\right)\left(\frac{\hat{\mathrm{Pr}}}{\mathrm{Pr}}\right) \frac{1}{\bar{c}^{2}(\gamma-1)}
$$

leads to

$$
V^{T} \mathcal{H}\left(B_{2} V-2 \epsilon G^{i}\right)=-\tilde{\phi}^{T} \mathcal{A} \tilde{\phi}+2 \hat{\kappa} \beta \tilde{T} \tilde{T}_{y}
$$

The temperature related terms in (18) and (37) can be written as vectormatrix multiplications

$$
\begin{aligned}
2 \bar{\kappa} \beta T T_{y} & =\left[\begin{array}{c}
T \\
\bar{\kappa} T_{y}
\end{array}\right]^{T}\left[\begin{array}{ll}
0 & \beta \\
\beta & 0
\end{array}\right]\left[\begin{array}{c}
T \\
\bar{\kappa} T_{y}
\end{array}\right], \\
2 \hat{\kappa} \beta \tilde{T} \tilde{T}_{y} & =\left[\begin{array}{c}
\tilde{T} \\
\hat{\kappa} \tilde{T}_{y}
\end{array}\right]^{T}\left[\begin{array}{ll}
0 & \beta \\
\beta & 0
\end{array}\right]\left[\begin{array}{c}
\tilde{T} \\
\hat{\kappa} \tilde{T}_{y}
\end{array}\right],
\end{aligned}
$$


or

$$
\begin{aligned}
2 \bar{\kappa} \beta T T_{y} & =\left[\begin{array}{c}
\bar{\kappa} T_{y}-\alpha T \\
\alpha T
\end{array}\right]^{T}\left[\begin{array}{cc}
-\beta / \alpha & 0 \\
0 & \beta / \alpha
\end{array}\right]\left[\begin{array}{c}
\bar{\kappa} T_{y}-\alpha T \\
\alpha T
\end{array}\right]+\frac{\beta \bar{\kappa}^{2} T_{y}^{2}}{\alpha}, \\
2 \hat{\kappa} \beta \tilde{T} \tilde{T}_{y} & =\left[\begin{array}{c}
-\alpha \tilde{T} \\
\hat{\kappa} \tilde{T}_{y}+\alpha \tilde{T}
\end{array}\right]^{T}\left[\begin{array}{cc}
-\beta / \alpha & 0 \\
0 & \beta / \alpha
\end{array}\right]\left[\begin{array}{c}
-\alpha \tilde{T} \\
\hat{\kappa} \tilde{T}_{y}+\alpha \tilde{T}
\end{array}\right]-\frac{\beta \hat{\kappa}^{2} \tilde{T}_{y}^{2}}{\alpha} .
\end{aligned}
$$

By using (38) and (39), we can rewrite the formulation (18) and (37) as

$$
\begin{aligned}
U^{T}\left(A_{2} U-2 \epsilon G^{c}\right) & =-\phi_{j}^{T} \mathcal{A}_{j} \phi_{j}-\delta_{2 j} \frac{\beta \bar{\kappa}^{2} T_{y}^{2}}{\alpha}, \\
V^{T} \mathcal{H}\left(B_{2} V-2 \epsilon G^{i}\right) & =-\tilde{\phi}_{j}^{T} \mathcal{A}_{j} \tilde{\phi}_{j}+\delta_{2 j} \frac{\beta \hat{\kappa}^{2} \tilde{T}_{y}^{2}}{\alpha},
\end{aligned}
$$

for $j=1,2$. The case $j=1$ is related to the conditions (25) and $j=2$ is related to the conditions (29). In (40), $\delta_{2 j}$ is the Kronecker delta, returning 1 if $j=2$ or 0 if $j=1$. The matrices $\mathcal{A}_{1}$ and $\mathcal{A}_{2}$ are

$$
\mathcal{A}_{1}=\left[\begin{array}{cccccc}
0 & 0 & 0 & \bar{\rho} & 0 & 0 \\
0 & 0 & -\bar{\rho} & 0 & 0 & 0 \\
0 & -\bar{\rho} & 0 & 0 & 0 & 0 \\
\bar{\rho} & 0 & 0 & 0 & 0 & 0 \\
0 & 0 & 0 & 0 & 0 & \beta \\
0 & 0 & 0 & 0 & \beta & 0
\end{array}\right], \quad \mathcal{A}_{2}=\left[\begin{array}{cccccc}
0 & 0 & 0 & \bar{\rho} & 0 & 0 \\
0 & 0 & -\bar{\rho} & 0 & 0 & 0 \\
0 & -\bar{\rho} & 0 & 0 & 0 & 0 \\
\bar{\rho} & 0 & 0 & 0 & 0 & 0 \\
0 & 0 & 0 & 0 & -\beta / \alpha & 0 \\
0 & 0 & 0 & 0 & 0 & \beta / \alpha
\end{array}\right] .
$$

By substituting the relations in (40) into (33), we obtain

$$
I T F=-\left.\int_{-L}^{+L}\left(\phi_{j}^{T} \mathcal{A}_{j} \phi_{j}-\tilde{\phi}_{j}^{T} \mathcal{A}_{j} \tilde{\phi}_{j}+\delta_{2 j} \frac{\beta}{\alpha}\left(\bar{\kappa}^{2} T_{y}^{2}+\hat{\kappa}^{2} \tilde{T}_{y}^{2}\right)\right)\right|_{y=0} d x .
$$

Inserting the interface conditions $\phi_{j}=\tilde{\phi}_{j}$ into (42) followed by time integration of (32) yields the estimate

$$
\begin{gathered}
\|U(T)\|_{2}^{2}+\|V(T)\|_{\mathcal{H} \tilde{I}_{4}}^{2}+2 \epsilon \int_{0}^{T}\left(D I S_{1}+\widetilde{D I S_{2}}\right) d t=\left\|f^{c}\right\|_{2}^{2}+\left\|f^{i}\right\|_{\mathcal{H}}^{2} \\
-\left.\delta_{2 j} \frac{\beta}{\alpha} \int_{0}^{T} \int_{-L}^{+L}\left(\bar{\kappa}^{2} T_{y}^{2}+\hat{\kappa}^{2} \tilde{T}_{y}^{2}\right)\right|_{y=0} d x d t
\end{gathered}
$$

where $f^{c}$ and $f^{i}$ are initial data for the systems (1) and (22), respectively.

Remark 13. The parameters related to the incompressible fluid are not involved in the matrices $\mathcal{A}_{1}$ and $\mathcal{A}_{2}$ due to the weights in (17) and (36). 


\subsection{Weakly imposed interface conditions}

In this subsection, we impose the interface conditions (25) or (29) weakly to the right-hand side of (1) and (22), as

$$
\begin{aligned}
U_{t}+A_{1} U_{x}+A_{2} U_{y} & =\epsilon\left(F_{x}^{c}+G_{y}^{c}\right)+L\left[\Sigma\left(\phi_{j}-\tilde{\phi}_{j}\right)\right], \\
\tilde{I}_{4} V_{t}+B_{1} V_{x}+B_{2} V_{y} & =\epsilon\left(F_{x}^{i}+G_{y}^{i}\right)+L\left[\tilde{\Sigma}\left(\tilde{\phi}_{j}-\phi_{j}\right)\right],
\end{aligned}
$$

for $j=1,2$. In (44), $L$ is a lifting operator [42] defined by $\int_{\Omega} \varphi^{T} L(\psi) d x d y=$ $\oint_{\partial \Omega} \varphi^{T} \psi d x d y$, for smooth vector functions $\varphi, \psi$ and domain $\Omega$ with boundary $\partial \Omega$. Furthermore $\Sigma, \tilde{\Sigma}$ are penalty matrices to be determined.

The energy method with the norm and weights given in (31), (17) and (36) applied to (44) leads to

$$
\frac{d}{d t}\left(\|U\|_{2}^{2}+\|V\|_{\mathcal{H} \tilde{I}_{4}}^{2}\right)+2 \epsilon D I S_{1}+2 \epsilon \widetilde{D I S}_{2}=I T F,
$$

where the interface term $I T F$ is

$$
\begin{aligned}
I T F & =\left.\int_{-L}^{+L}\left(U^{T}\left(A_{2} U-2 \epsilon G^{c}\right)-V^{T} \mathcal{H}\left(B_{2} V-2 \epsilon G^{i}\right)\right)\right|_{y=0} d x \\
& +\left.\int_{-L}^{+L}\left(U^{T} \Sigma\left(\phi_{j}-\tilde{\phi}_{j}\right)+\left(U^{T} \Sigma\left(\phi_{j}-\tilde{\phi}_{j}\right)\right)^{T}\right)\right|_{y=0} d x \\
& +\left.\int_{-L}^{+L}\left(V^{T} \mathcal{H} \tilde{\Sigma}\left(\tilde{\phi}_{j}-\phi_{j}\right)+\left(V^{T} \mathcal{H} \tilde{\Sigma}\left(\tilde{\phi}_{j}-\phi_{j}\right)\right)^{T}\right)\right|_{y=0} d x
\end{aligned}
$$

By substituting the relation (40) into (45), we get

$$
\begin{aligned}
I T F= & -\left.\int_{-L}^{+L}\left(\phi_{j}^{T} \mathcal{A}_{j} \phi_{j}-\tilde{\phi}_{j}^{T} \mathcal{A}_{j} \tilde{\phi}_{j}+\delta_{2 j} \frac{\beta}{\alpha}\left(\bar{\kappa}^{2} T_{y}^{2}+\hat{\kappa}^{2} \tilde{T}_{y}^{2}\right)\right)\right|_{y=0} d x \\
& +\left.\int_{-L}^{+L}\left(U^{T} \Sigma\left(\phi_{j}-\tilde{\phi}_{j}\right)+\left(U^{T} \Sigma\left(\phi_{j}-\tilde{\phi}_{j}\right)\right)^{T}\right)\right|_{y=0} d x \\
& +\left.\int_{-L}^{+L}\left(V^{T} \mathcal{H} \tilde{\Sigma}\left(\tilde{\phi}_{j}-\phi_{j}\right)+\left(V^{T} \mathcal{H} \tilde{\Sigma}\left(\tilde{\phi}_{j}-\phi_{j}\right)\right)^{T}\right)\right|_{y=0} d x
\end{aligned}
$$

Further, we mimic the analysis in [10] and let

$$
\Sigma=H^{T} \Sigma_{c}, \quad \tilde{\Sigma}=\mathcal{H}^{-1} \tilde{H}^{T} \Sigma_{i},
$$


where the differential operators $H$ and $\tilde{H}$ are given in (26) and (30) and $\Sigma_{c}$ and $\Sigma_{i}$ are matrices of size $6 \times 6$. Using (47) leads to

$$
\begin{aligned}
I T F= & -\left.\int_{-L}^{+L}\left(\phi_{j}^{T} \mathcal{A}_{j} \phi_{j}-\tilde{\phi}_{j}^{T} \mathcal{A}_{j} \tilde{\phi}_{j}+\delta_{2 j} \frac{\beta}{\alpha}\left(\bar{\kappa}^{2} T_{y}^{2}+\hat{\kappa}^{2} \tilde{T}_{y}^{2}\right)\right)\right|_{y=0} d x \\
& +\left.\int_{-L}^{+L}\left(\phi_{j}^{T} \Sigma_{c}\left(\phi_{j}-\tilde{\phi}_{j}\right)+\left(\phi_{j}^{T} \Sigma_{c}\left(\phi_{j}-\tilde{\phi}_{j}\right)\right)^{T}\right)\right|_{y=0} d x \\
& +\left.\int_{-L}^{+L}\left(\tilde{\phi}_{j}^{T} \Sigma_{i}\left(\tilde{\phi}_{j}-\phi_{j}\right)+\left(\tilde{\phi}_{j}^{T} \Sigma_{i}\left(\tilde{\phi}_{j}-\phi_{j}\right)\right)^{T}\right)\right|_{y=0} d x \\
= & -\left.\int_{-L}^{+L}\left[\begin{array}{c}
\phi_{j} \\
\tilde{\phi}_{j}
\end{array}\right]^{T}\left[\begin{array}{cc}
\mathcal{A}_{j}-\Sigma_{c}-\Sigma_{c}^{T} & \Sigma_{c}+\Sigma_{i}^{T} \\
\Sigma_{c}^{T}+\Sigma_{i} & -\mathcal{A}_{j}-\Sigma_{i}-\Sigma_{i}^{T}
\end{array}\right]\left[\begin{array}{c}
\phi_{j} \\
\tilde{\phi}_{j}
\end{array}\right]\right|_{y=0} d x \\
& -\left.\delta_{2 j} \frac{\beta}{\alpha} \int_{-L}^{+L}\left(\bar{\kappa}^{2} T_{y}^{2}+\hat{\kappa}^{2} \tilde{T}_{y}^{2}\right)\right|_{y=0} d x .
\end{aligned}
$$

Next, we choose $\Sigma_{c}=\mathcal{A}_{j}+\Sigma_{i}$ and rewrite (48) as

$$
\begin{aligned}
I T F= & -\left.\int_{-L}^{+L}\left[\begin{array}{c}
\phi_{j} \\
\tilde{\phi}_{j}
\end{array}\right]^{T}\left(C \otimes\left(\mathcal{A}_{j}+\Sigma_{i}+\Sigma_{i}^{T}\right)\right)\left[\begin{array}{c}
\phi_{j} \\
\tilde{\phi}_{j}
\end{array}\right]\right|_{y=0} d x \\
& -\left.\delta_{2 j} \frac{\beta}{\alpha} \int_{-L}^{+L}\left(\bar{\kappa}^{2} T_{y}^{2}+\hat{\kappa}^{2} \tilde{T}_{y}^{2}\right)\right|_{y=0} d x, C=\left[\begin{array}{cc}
-1 & 1 \\
1 & -1
\end{array}\right],
\end{aligned}
$$

where $\otimes$ denotes the Kronecker product [43].

Since the matrix $C$ is negative semi-definite, we must choose $\Sigma_{i}$ such that $\mathcal{A}_{j}+\Sigma_{i}+\Sigma_{i}^{T} \leq 0$. The matrix $\mathcal{A}_{j}$ can be split as $\mathcal{A}_{j}=\mathcal{A}_{j}^{+}+\mathcal{A}_{j}^{-}$, where $\mathcal{A}_{j}^{+}=X_{+}^{j} \Lambda_{j}^{+}\left(X_{+}^{j}\right)^{T}, \mathcal{A}_{j}^{-}=X_{-}^{j} \Lambda_{j}^{-}\left(X_{-}^{j}\right)^{T}$ and $\Lambda_{j}^{+}, \Lambda_{j}^{-}, X_{+}^{j}, X_{-}^{j}$ contain the positive and negative eigenvalues of $\mathcal{A}_{j}$ and the corresponding eigenvectors respectively. The specific choice $\Sigma_{i}=-\mathcal{A}_{j}^{+} / 2$ leads to

$$
\begin{aligned}
\frac{d}{d t}\left(\|U\|_{2}^{2}+\|V\|_{\mathcal{H} \tilde{I}_{4}}^{2}\right)+2 \epsilon D I S_{1}+2 \epsilon \widetilde{D I S_{2}} & =\left.\int_{-L}^{+L}\left(\phi_{j}-\tilde{\phi}_{j}\right)^{T} \mathcal{A}_{j}^{-}\left(\phi_{j}-\tilde{\phi}_{j}\right)\right|_{y=0} d x \\
& -\left.\delta_{2 j} \frac{\beta}{\alpha} \int_{-L}^{+L}\left(\bar{\kappa}^{2} T_{y}^{2}+\hat{\kappa}^{2} \tilde{T}_{y}^{2}\right)\right|_{y=0} d x .
\end{aligned}
$$


Time integration of (49) yields

$$
\begin{aligned}
\|U(T)\|_{2}^{2}+\|V(T)\|_{\mathcal{H} \tilde{I}_{4}}^{2} & +2 \epsilon \int_{0}^{T}\left(D I S_{1}+\widetilde{D I S_{2}}\right) d t=\left\|f^{c}\right\|_{2}^{2}+\left\|f^{i}\right\|_{\mathcal{H}}^{2} \\
& +\left.\int_{0}^{T} \int_{-L}^{+L}\left(\phi_{j}-\tilde{\phi}_{j}\right)^{T} \mathcal{A}_{j}^{-}\left(\phi_{j}-\tilde{\phi}_{j}\right)\right|_{y=0} d x d t \\
& -\left.\delta_{2 j} \frac{\beta}{\alpha} \int_{0}^{T} \int_{-L}^{+L}\left(\bar{\kappa}^{2} T_{y}^{2}+\hat{\kappa}^{2} \tilde{T}_{y}^{2}\right)\right|_{y=0} d x d t
\end{aligned}
$$

Remark 14. The choices for the penalty matrices, i.e. $\Sigma_{c}=\mathcal{A}_{j}+\Sigma_{i}$ and $\Sigma_{i}=-\mathcal{A}_{j}^{+} / 2$ are not unique, other choices can also be made.

Remark 15. Comparing the energy estimates in (43) with (50) shows that the weak imposition produces the same energy rate as the strong imposition with an additional damping term. A similar term will appear in the discrete approximation.

We summarize the result in

Proposition 2. The weak imposition of either the interface conditions in (25) with the penalty matrices

$$
\Sigma=H^{T}\left(\mathcal{A}_{1}-\frac{\mathcal{A}_{1}^{+}}{2}\right), \quad \tilde{\Sigma}=-\mathcal{H}^{-1} \tilde{H}^{T} \frac{\mathcal{A}_{1}^{+}}{2} .
$$

or the conditions in (29) with the penalty matrices

$$
\Sigma=H^{T}\left(\mathcal{A}_{2}-\frac{\mathcal{A}_{2}^{+}}{2}\right), \quad \tilde{\Sigma}=-\mathcal{H}^{-1} \tilde{H}^{T} \frac{\mathcal{A}_{2}^{+}}{2} .
$$

leads to an energy estimate.

\section{The discrete problem}

In this section, we follow the procedure in $[9,10]$ and show that the continuous analysis above lead directly to stability for SBP based schemes $[11,12,13,14,15,16,17]$. To discretize the coupled problem (44), finite differences on SBP form $[44,45,46]$ are used. The interface conditions are implemented weakly using simultaneous approximation terms (SAT) [31, 47, 48]. We consider the computational domains $\Omega_{i}$ and $\Omega_{c}$ with meshes using 
$(N+1) \times(M+1)$ points. For simplicity we use collocated nodes (noncollocated are also possible $[49,50])$. We also use $K+1$ time levels from 0 to $T$. Since there is no time derivate for pressure in the incompressible equations on velocity-divergence form, implicit methods are suitable for time discretization. In this paper, we use the high order accurate SBP-SAT finite difference technique also in time.

Let the vectors $\mathbf{U}=\left\{\mathbf{U}_{0}, \cdots, \mathbf{U}_{K}\right\}$ and $\mathbf{V}=\left\{\mathbf{V}_{0}, \cdots, \mathbf{V}_{K}\right\}$ with length $(K+1) \times(N+1) \times(M+1)$ contain the discrete approximations of the solutions, where

$$
\begin{aligned}
& \mathbf{U}_{k}=\left\{U_{k 00}, \cdots, U_{k 0 M}, U_{k 10}, \cdots, U_{k 1 M}, \cdots, U_{k N 0}, \cdots, U_{k N M}\right\}, U_{k i j} \approx U\left(t_{k}, x_{i}, y_{j}\right), \\
& \mathbf{V}_{k}=\left\{V_{k 00}, \cdots, V_{k 0 M}, V_{k 10}, \cdots, V_{k 1 M}, \cdots, V_{k N 0}, \cdots, V_{k N M}\right\}, \quad V_{k i j} \approx V\left(t_{k}, x_{i}, y_{j}\right) .
\end{aligned}
$$

The first derivatives of $U$ with respect to $t, x$ and $y$ are approximated by

$$
\begin{aligned}
\mathbf{U}_{t}=\mathcal{D}_{t} \mathbf{U} \approx U_{t}, & \mathcal{D}_{t}=P_{t}^{-1} Q_{t} \otimes I_{x} \otimes I_{y} \otimes I_{4}, \\
\mathbf{U}_{x}=\mathcal{D}_{x} \mathbf{U} \approx U_{x}, & \mathcal{D}_{x}=I_{t} \otimes P_{x}^{-1} Q_{x} \otimes I_{y} \otimes I_{4}, \\
\mathbf{U}_{y}=\mathcal{D}_{y} \mathbf{U} \approx U_{y}, & \mathcal{D}_{y}=I_{t} \otimes I_{x} \otimes P_{y}^{-1} Q_{y} \otimes I_{4},
\end{aligned}
$$

where $I_{t}, I_{x}, I_{y}$ and $I_{4}$ are identity matrices with size $K+1, N+1, M+1$ and 4 , respectively. The matrices $P_{t, x, y}$ are symmetric positive definite, and $Q_{t, x, y}$ are almost skew-symmetric matrices, satisfying

$$
Q_{t}+Q_{t}^{T}=E_{K}-E_{0}, \quad Q_{x}+Q_{x}^{T}=E_{N}-E_{0}, \quad Q_{y}+Q_{y}^{T}=E_{M}-E_{0} .
$$

In (53), $E_{0}=\operatorname{diag}(1,0, \cdots, 0)$ and $E_{K, N, M}=\operatorname{diag}(0, \cdots, 0,1)$ with the appropriate dimensions. The derivatives of $V$ are approximated in a similar way.

An SBP-SAT discretization of (44) can be written as

$$
\begin{aligned}
& \mathcal{D}_{t} \mathbf{U}+\left[D_{x} \otimes A_{1}+D_{y} \otimes A_{2}\right] \mathbf{U}-\epsilon\left(\mathcal{D}_{x} \mathbf{F}^{c}+\mathcal{D}_{y} \mathbf{G}^{c}\right)=\mathbb{S}+\mathbb{S}_{t}, \\
& \tilde{\mathcal{D}}_{t} \mathbf{V}+\left[D_{x} \otimes B_{1}+D_{y} \otimes B_{2}\right] \mathbf{V}-\epsilon\left(\mathcal{D}_{x} \mathbf{F}^{i}+\mathcal{D}_{y} \mathbf{G}^{i}\right)=\tilde{\mathbb{S}}+\tilde{\mathbb{S}}_{t},
\end{aligned}
$$

where $D_{x}=I_{t} \otimes P_{x}^{-1} Q_{x} \otimes I_{y}, D_{y}=I_{t} \otimes I_{x} \otimes P_{y}^{-1} Q_{y}, \tilde{\mathcal{D}}_{t}=P_{t}^{-1} Q_{t} \otimes I_{x} \otimes I_{y} \otimes \tilde{I}_{4}$ and

$$
\begin{array}{ll}
\mathbf{F}^{c}=\left(I \otimes A_{11}\right) \mathbf{U}_{x}+\left(I \otimes A_{12}\right) \mathbf{U}_{y}, & \mathbf{G}^{c}=\left(I \otimes A_{21}\right) \mathbf{U}_{x}+\left(I \otimes A_{22}\right) \mathbf{U}_{y}, \\
\mathbf{F}^{i}=\left(I \otimes B_{11}\right) \mathbf{V}_{x}+\left(I \otimes B_{12}\right) \mathbf{V}_{y}, & \mathbf{G}^{i}=\left(I \otimes B_{21}\right) \mathbf{V}_{x}+\left(I \otimes B_{22}\right) \mathbf{V}_{y},
\end{array}
$$


with $I=I_{t} \otimes I_{x} \otimes I_{y}$. For simplicity, we use the first derivative twice as our second derivative operator. The outer boundary conditions in (54) are ignored as in the continuous case. Moreover, $\mathbb{S}$ and $\tilde{\mathbb{S}}$ in $(54)$, are the discrete lifting operators corresponding to (44), and given by

$$
\begin{aligned}
& \mathbb{S}=\left(I_{t} \otimes I_{x} \otimes P_{y}^{-1} E_{0} \otimes I_{4}\right) \boldsymbol{\Sigma}\left(\boldsymbol{\phi}_{j}-\tilde{\boldsymbol{\phi}}_{j}\right), \\
& \tilde{\mathbb{S}}=\left(I_{t} \otimes I_{x} \otimes P_{y}^{-1} E_{M} \otimes I_{4}\right) \tilde{\Sigma}\left(\tilde{\boldsymbol{\phi}}_{j}-\boldsymbol{\phi}_{j}\right) .
\end{aligned}
$$

In (55),

$$
\boldsymbol{\phi}_{j}=\mathbf{H U}, \quad \tilde{\boldsymbol{\phi}}_{j}=\tilde{\mathbf{H}} \mathbf{V} .
$$

where the matrices $\mathbf{H}$ and $\tilde{\mathbf{H}}$ are discrete versions of $H$ and $\tilde{H}$ either in (26) or (30), given by

$$
\begin{aligned}
\mathbf{H} & =\left(I \otimes H_{0}\right)+\left(D_{x} \otimes H_{x}\right)+\left(D_{y} \otimes H_{y}\right), \\
\tilde{\mathbf{H}} & =\left(I \otimes \tilde{H}_{0}\right)+\left(D_{x} \otimes \tilde{H}_{x}\right)+\left(D_{y} \otimes \tilde{H}_{y}\right) .
\end{aligned}
$$

Finally, $\mathbb{S}_{t}$ and $\tilde{\mathbb{S}}_{t}$ are the penalty terms for weakly imposing the initial conditions given by

$$
\begin{aligned}
& \mathbb{S}_{t}=\left(P_{t}^{-1} E_{0} \otimes I_{x} \otimes I_{y} \otimes I_{4}\right) \Sigma_{t}^{c}\left(\mathbf{U}-\mathbf{f}^{c}\right), \\
& \tilde{\mathbb{S}}_{t}=\left(P_{t}^{-1} E_{0} \otimes I_{x} \otimes I_{y} \otimes I_{4}\right) \Sigma_{t}^{i}\left(\mathbf{V}-\mathbf{f}^{i}\right),
\end{aligned}
$$

where $\mathbf{f}^{c}=E_{0} \otimes I_{x} \otimes I_{y} \otimes f_{c}$ and $\mathbf{f}^{i}=E_{0} \otimes I_{x} \otimes I_{y} \otimes f_{i}$ containing the initial data for the upper and lower domain, respectively.

\subsection{Stability}

The SBP-SAT scheme is constructed such that it mimics the continuous integration-by-parts based energy analysis performed in Subsection 3.3. We will take advantage of that and multiply the discrete problems (54) with $\mathbf{U}^{T}\left(P_{t} \otimes P_{x} \otimes P_{y} \otimes I_{4}\right)$ and $\mathbf{V}^{T}\left(P_{t} \otimes P_{x} \otimes P_{y} \otimes \mathcal{H}\right)$, respectively (i.e. using the discrete energy method). The matrix $\mathcal{H}$ is defined in (31) with the weights given in (17) and (36). We add the results to their transpose and use the SBP properties (53) to arrive at

$$
\left\|\mathbf{U}_{K}\right\|_{P_{x y} \otimes I_{4}}^{2}+\left\|\mathbf{V}_{K}\right\|_{P_{x y} \otimes \mathcal{H} \tilde{I}_{4}}^{2}+2 \epsilon \mathbf{D I S}_{1}+2 \epsilon \widetilde{\mathbf{D I S}}{ }_{2}=\mathbf{I T F}+\mathbf{I T},
$$


where $\left\|\mathbf{U}_{k}\right\|_{P_{x y} \otimes I_{4}}^{2}=\mathbf{U}_{k}^{T}\left(P_{x} \otimes P_{y} \otimes I_{4}\right) \mathbf{U}_{k}$ and $\left\|\mathbf{V}_{k}\right\|_{P_{x y} \otimes \mathcal{H} \tilde{I}_{4}}^{2}=\mathbf{V}_{k}^{T}\left(P_{x} \otimes P_{y} \otimes\right.$ $\left.\mathcal{H} \tilde{I}_{4}\right) \mathbf{V}_{k}, k=0, K$, are the discrete norms at the first and last time level. The discrete dissipation terms in (56) are

$$
\mathbf{D I S}_{1}=\left[\begin{array}{l}
\mathbf{U}_{x} \\
\mathbf{U}_{y}
\end{array}\right]^{T}\left(P_{t x y} \otimes D_{1}\right)\left[\begin{array}{l}
\mathbf{U}_{x} \\
\mathbf{U}_{y}
\end{array}\right] \geq 0, \widetilde{\mathbf{D I S}}{ }_{2}=\left[\begin{array}{c}
\mathbf{V}_{x} \\
\mathbf{V}_{y}
\end{array}\right]^{T}\left(P_{t x y} \otimes \tilde{D}_{2}\right)\left[\begin{array}{c}
\mathbf{V}_{x} \\
\mathbf{V}_{y}
\end{array}\right] \geq 0
$$

where the matrices $D_{1}$ and $\tilde{D}_{2}$ are given in (8) and (34), respectively and $P_{t x y}=P_{t} \otimes P_{x} \otimes P_{y}$.

The discrete interface term ITF corresponds to (45) in the continuous case and is given by

$$
\begin{aligned}
& \mathbf{I T F}=\mathbf{U}^{T}\left(P_{t} \otimes P_{x} \otimes E_{0} \otimes A_{2}\right) \mathbf{U}-2 \epsilon \mathbf{U}^{T}\left(P_{t} \otimes P_{x} \otimes E_{0} \otimes I_{4}\right) \mathbf{G}^{c} \\
& \quad+\mathbf{U}^{T}\left(P_{t} \otimes P_{x} \otimes E_{0} \otimes I_{4}\right) \boldsymbol{\Sigma}\left(\boldsymbol{\phi}_{j}-\tilde{\boldsymbol{\phi}}_{j}\right)+\left(\mathbf{U}^{T}\left(P_{t} \otimes P_{x} \otimes E_{0} \otimes I_{4}\right) \boldsymbol{\Sigma}\left(\boldsymbol{\phi}_{j}-\tilde{\boldsymbol{\phi}}_{j}\right)\right)^{T} \\
& \quad-\mathbf{V}^{T}\left(P_{t} \otimes P_{x} \otimes E_{M} \otimes \mathcal{H} B_{2}\right) \mathbf{V}+2 \epsilon \mathbf{V}^{T}\left(P_{t} \otimes P_{x} \otimes E_{M} \otimes \mathcal{H}\right) \mathbf{G}^{i} \\
& \quad+\mathbf{V}^{T}\left(P_{t} \otimes P_{x} \otimes E_{M} \otimes \mathcal{H}\right) \tilde{\boldsymbol{\Sigma}}\left(\tilde{\boldsymbol{\phi}}_{j}-\boldsymbol{\phi}_{j}\right)+\left(\mathbf{V}^{T}\left(P_{t} \otimes P_{x} \otimes E_{M} \otimes \mathcal{H}\right) \tilde{\boldsymbol{\Sigma}}\left(\tilde{\boldsymbol{\phi}}_{j}-\boldsymbol{\phi}_{j}\right)\right)^{T} .
\end{aligned}
$$

Furthermore, the penalty term for imposing the initial conditions is

$$
\begin{aligned}
\mathbf{I T} & =\mathbf{U}\left(E_{0} \otimes P_{x} \otimes P_{y} \otimes I_{4}\right) \Sigma_{t}^{c}\left(\mathbf{U}-\mathbf{f}^{c}\right)+\left(\mathbf{U}\left(E_{0} \otimes P_{x} \otimes P_{y} \otimes I_{4}\right) \Sigma_{t}^{c}\left(\mathbf{U}-\mathbf{f}^{c}\right)\right)^{T} \\
& +\mathbf{V}\left(E_{0} \otimes P_{x} \otimes P_{y} \otimes \mathcal{H}\right) \Sigma_{t}^{i}\left(\mathbf{V}-\mathbf{f}^{i}\right)+\left(\mathbf{V}\left(E_{0} \otimes P_{x} \otimes P_{y} \otimes \mathcal{H}\right) \Sigma_{t}^{c}\left(\mathbf{V}-\mathbf{f}^{i}\right)\right)^{T} \\
& +\left\|\mathbf{U}_{0}\right\|_{P_{x y} \otimes I_{4}}^{2}+\left\|\mathbf{V}_{0}\right\|_{P_{x y} \otimes \mathcal{H} \tilde{I}_{4}}^{2} .
\end{aligned}
$$

By using (40), we can rewrite (57) as

$$
\begin{aligned}
\mathbf{I T F} & =-\left(\boldsymbol{\phi}_{j}^{0}\right)^{T} \mathbf{A}_{j} \boldsymbol{\phi}_{j}^{0}+\left(\tilde{\boldsymbol{\phi}}_{j}^{M}\right)^{T} \mathbf{A}_{j} \tilde{\boldsymbol{\phi}}_{j}^{M} \\
& -\delta_{2 j} \frac{\beta}{\alpha}\left(\bar{\kappa}^{2}\left(\mathbf{T}_{y}^{0}\right)^{T} P_{t x} \mathbf{T}_{y}^{0}+\hat{\kappa}^{2}\left(\tilde{\mathbf{T}}_{y}^{M}\right)^{T} P_{t x} \tilde{\mathbf{T}}_{y}^{M}\right) \\
& +\mathbf{U}^{T}\left(P_{t} \otimes P_{x} \otimes E_{0} \otimes I_{4}\right) \boldsymbol{\Sigma}\left(\boldsymbol{\phi}_{j}-\tilde{\boldsymbol{\phi}}_{j}\right)+\left(\mathbf{U}^{T}\left(P_{t} \otimes P_{x} \otimes E_{0} \otimes I_{4}\right) \boldsymbol{\Sigma}\left(\boldsymbol{\phi}_{j}-\tilde{\boldsymbol{\phi}}_{j}\right)\right)^{T} \\
& +\mathbf{V}^{T}\left(P_{t} \otimes P_{x} \otimes E_{M} \otimes \mathcal{H}\right) \tilde{\Sigma}\left(\tilde{\boldsymbol{\phi}}_{j}-\boldsymbol{\phi}_{j}\right)+\left(\mathbf{V}^{T}\left(P_{t} \otimes P_{x} \otimes E_{M} \otimes \mathcal{H}\right) \tilde{\boldsymbol{\Sigma}}\left(\tilde{\boldsymbol{\phi}}_{j}-\boldsymbol{\phi}_{j}\right)\right)^{T},
\end{aligned}
$$

which corresponds to (46). In (58),

$$
\begin{array}{ll}
\mathbf{T}_{y}^{0}=\left(I_{t} \otimes I_{x} \otimes e_{0}\right) \mathbf{T}_{y}, & \tilde{\mathbf{T}}_{y}^{M}=\left(I_{t} \otimes I_{x} \otimes e_{M}\right) \tilde{\mathbf{T}}_{y}, \\
\boldsymbol{\phi}_{j}^{0}=\left(I_{t} \otimes I_{x} \otimes e_{0} \otimes I_{6}\right) \boldsymbol{\phi}_{j}, & \tilde{\boldsymbol{\phi}}_{j}^{M}=\left(I_{t} \otimes I_{x} \otimes e_{M} \otimes I_{6}\right) \tilde{\boldsymbol{\phi}}_{j},
\end{array}
$$


and

$$
\mathbf{T}_{y}=D_{y} \mathbf{T}, \quad \tilde{\mathbf{T}}_{y}=D_{y} \tilde{\mathbf{T}}, \quad \mathbf{A}_{j}=\left(P_{t x} \otimes \mathcal{A}_{j}\right), \quad P_{t x}=P_{t} \otimes P_{x},
$$

with $\mathcal{A}_{j}$ given in (41). The row vectors $e_{0}$ and $e_{M}$ are zero except for the first and last element respectively which is one.

We follow a similar procedure as in the continuous case and let $\Sigma=$ $\mathbf{H}^{T} \boldsymbol{\Sigma}_{c}, \boldsymbol{\Sigma}_{c}=\left(I \otimes \Sigma_{c}\right)$ and $\tilde{\boldsymbol{\Sigma}}=\left(I \otimes \mathcal{H}^{-1}\right) \tilde{\mathbf{H}}^{T} \boldsymbol{\Sigma}_{i}, \boldsymbol{\Sigma}_{i}=\left(I \otimes \Sigma_{i}\right)$. Inserting these penalty matrices into (58) leads to

$$
\begin{aligned}
\mathbf{I T F}= & -\left[\begin{array}{c}
\boldsymbol{\phi}_{j}^{0} \\
\tilde{\boldsymbol{\phi}}_{j}^{M}
\end{array}\right]^{T}\left(P_{t x} \otimes\left[\begin{array}{cc}
\mathcal{A}_{j}-\Sigma_{c}-\Sigma_{c}^{T} & \Sigma_{c}+\Sigma_{i}^{T} \\
\Sigma_{c}^{T}+\Sigma_{i} & -\mathcal{A}_{j}-\Sigma_{i}-\Sigma_{i}^{T}
\end{array}\right]\right)\left[\begin{array}{c}
\boldsymbol{\phi}_{j}^{0} \\
\tilde{\boldsymbol{\phi}}_{j}^{M}
\end{array}\right] \\
& -\delta_{2 j} \frac{\beta}{\alpha}\left(\bar{\kappa}^{2}\left(\mathbf{T}_{y}^{0}\right)^{T} P_{t x} \mathbf{T}_{y}^{0}+\hat{\kappa}^{2}\left(\tilde{\mathbf{T}}_{y}^{M}\right)^{T} P_{t x} \tilde{\mathbf{T}}_{y}^{M}\right)
\end{aligned}
$$

corresponding to (48). Choosing $\Sigma_{i}$ and $\Sigma_{c}$ as in (51) or (52) in the continuous case, yields

$$
\begin{aligned}
\mathbf{I T F} & =\left(\boldsymbol{\phi}_{j}^{0}-\tilde{\boldsymbol{\phi}}_{j}^{M}\right)^{T}\left(P_{t x} \otimes \mathcal{A}_{j}^{-}\right)\left(\boldsymbol{\phi}_{j}^{0}-\tilde{\boldsymbol{\phi}}_{j}^{M}\right) \\
& -\delta_{2 j} \frac{\beta}{\alpha}\left(\bar{\kappa}^{2}\left(\mathbf{T}_{y}^{0}\right)^{T} P_{t x} \mathbf{T}_{y}^{0}+\hat{\kappa}^{2}\left(\tilde{\mathbf{T}}_{y}^{M}\right)^{T} P_{t x} \tilde{\mathbf{T}}_{y}^{M}\right),
\end{aligned}
$$

which is the discrete analogue of right-hand side of (49).

Next, we consider the terms related to the initial condition. Let $\Sigma_{t}^{c}=$ $I \otimes \Sigma_{t}^{c}$ and $\Sigma_{t}^{i}=I \otimes \Sigma_{t}^{i}$, where $\Sigma_{t}^{c}$ and $\Sigma_{t}^{i}$ are $4 \times 4$ matrices. Then, the initial term IT can be rewritten as

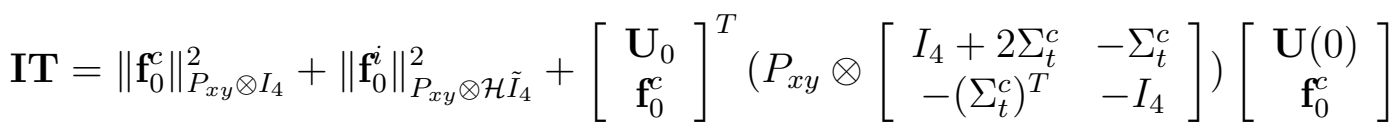

$$
\begin{aligned}
& +\left[\begin{array}{c}
\mathbf{V}_{0} \\
\mathbf{f}_{0}^{i}
\end{array}\right]^{T}\left(P_{x y} \otimes\left[\begin{array}{cc}
\mathcal{H} \tilde{I}_{4}+2 \mathcal{H} \Sigma_{t}^{i} & -\mathcal{H} \Sigma_{t}^{i} \\
-\mathcal{H}\left(\Sigma_{t}^{i}\right)^{T} & -\mathcal{H} \tilde{I}_{4}
\end{array}\right]\right)\left[\begin{array}{c}
\mathbf{V}_{0} \\
\mathbf{f}_{0}^{i}
\end{array}\right], \quad P_{x y}=P_{x} \otimes P_{y}
\end{aligned}
$$

where $\mathbf{f}_{0}^{c}=I_{x} \otimes I_{y} \otimes f^{c}, \mathbf{f}_{0}^{i}=I_{x} \otimes I_{y} \otimes f^{i}$ and we have added and subtracted $\left\|\mathbf{f}_{0}^{c}\right\|_{P_{x y} \otimes I_{4}}^{2}+\left\|\mathbf{f}_{0}^{i}\right\|_{P_{x y} \otimes \mathcal{H} \tilde{I}_{4}}^{2}$. Choosing $\Sigma_{t}^{c}=-I_{4}$ and $\Sigma_{t}^{i}=-\tilde{I}_{4}$ yields

$$
\mathbf{I T}=\left\|\mathbf{f}_{0}^{\mathbf{c}}\right\|_{P_{x y} \otimes I_{4}}^{2}+\left\|\mathbf{f}_{0}^{i}\right\|_{P_{x y} \otimes \mathcal{H} \tilde{I}_{4}}^{2}-\left\|\mathbf{U}_{0}-\mathbf{f}_{0}^{c}\right\|_{P_{x y} \otimes I_{4}}^{2}-\left\|\mathbf{V}_{0}-\mathbf{f}_{0}^{i}\right\|_{P_{x y} \otimes \mathcal{H} \tilde{I}_{4}}^{2} .
$$


Now, by substituting (59) and (60) into (56), we get

$$
\begin{aligned}
& \left\|\mathbf{U}_{K}\right\|_{P_{x y} \otimes I_{4}}^{2}+\left\|\mathbf{V}_{K}\right\|_{P_{x y} \otimes \mathcal{H} \tilde{I}_{4}}^{2}+2 \epsilon \mathbf{D I} \mathbf{S}_{1}+2 \epsilon \widetilde{\mathbf{D I S}}{ }_{2}=\left\|\mathbf{f}_{0}^{c}\right\|_{P_{x y} \otimes I_{4}}^{2}+\left\|\mathbf{f}_{0}^{i}\right\|_{P_{x y} \otimes \mathcal{H} \tilde{I}_{4}}^{2} \\
& -\left\|\mathbf{U}_{0}-\mathbf{f}_{0}^{c}\right\|_{P_{x y} \otimes I_{4}}^{2}-\left\|\mathbf{V}_{0}-\mathbf{f}_{0}^{i}\right\|_{P_{x y} \otimes \mathcal{H} \tilde{I}_{4}}^{2}+\left(\boldsymbol{\phi}_{j}^{0}-\tilde{\boldsymbol{\phi}}_{j}^{M}\right)^{T}\left(P_{t x} \otimes \mathcal{A}_{j}^{-}\right)\left(\boldsymbol{\phi}_{j}^{0}-\tilde{\boldsymbol{\phi}}_{j}^{M}\right) \\
& -\delta_{2 j} \frac{\beta}{\alpha}\left(\bar{\kappa}^{2}\left(\mathbf{T}_{y}^{0}\right)^{T} P_{t x} \mathbf{T}_{y}^{0}+\hat{\kappa}^{2}\left(\tilde{\mathbf{T}}_{y}^{M}\right)^{T} P_{t x} \tilde{\mathbf{T}}_{y}^{M}\right),
\end{aligned}
$$

which is the discrete version of the continuous estimate (50) with additional damping terms due to the weak imposition of the initial conditions. We summarize the result of this section in the following proposition.

Proposition 3. The scheme (54) with either penalty matrices

$$
\boldsymbol{\Sigma}=\boldsymbol{H}^{T}\left(I \otimes\left(\mathcal{A}_{1}-\frac{\mathcal{A}_{1}^{+}}{2}\right)\right), \tilde{\Sigma}=-\left(I \otimes \mathcal{H}^{-1}\right) \tilde{\boldsymbol{H}}^{T}\left(I \otimes \frac{\mathcal{A}_{1}^{+}}{2}\right),
$$

or

$$
\Sigma=\boldsymbol{H}^{T}\left(I \otimes\left(\mathcal{A}_{2}-\frac{\mathcal{A}_{2}^{+}}{2}\right)\right), \tilde{\Sigma}=-\left(I \otimes \mathcal{H}^{-1}\right) \tilde{\boldsymbol{H}}^{T}\left(I \otimes \frac{\mathcal{A}_{2}^{+}}{2}\right),
$$

and $\Sigma_{t}^{c}=-I \otimes I_{4}, \Sigma_{t}^{i}=-\tilde{I} \otimes I_{4}$ is stable.

Remark 16. Proposition 3 is the fully discrete version of Proposition 2.

\section{Numerical results}

In this section we investigate the accuracy of our numerical scheme using the method of manufactured solutions [51]. The manufactured solution is

$$
\begin{aligned}
\rho & =1+\frac{1}{2} \sin (\pi(x-y)-t), & u & =\frac{1}{2} \cos (\pi x+y-t), \\
v & =\frac{1}{2} \sin (x+\pi y-t), & p & =1+\frac{1}{2} \sin (\pi(x+y)-t), \\
\tilde{u} & =\frac{1}{2} \cos (\pi x+y-t), & \tilde{v} & =\frac{1}{2} \sin (x+\pi y-t), \\
\tilde{p} & =1+\frac{1}{2} \sin (\pi(x+y)-t), & \tilde{T} & =1+\frac{1}{2} \sin (\pi(x-y)-t) .
\end{aligned}
$$

The initial conditions $U=f^{c}, V=f^{i}$ and the far-field boundary conditions derived in [52] are used. The manufactured solution (61) is the solution to the modified systems

$$
\begin{aligned}
U_{t}+A_{1} U_{x}+A_{2} U_{y} & =\epsilon\left(F_{x}^{c}+G_{y}^{c}\right)+F \\
\tilde{I}_{4} V_{t}+B_{1} V_{x}+B_{2} V_{y} & =\epsilon\left(F_{x}^{i}+G_{y}^{i}\right)+G,
\end{aligned}
$$


where the forcing functions $F$ and $G$ are computed by using (61). The functions in (61) satisfy the interface conditions in a non-trivial way.

The rates of convergence are computed as

$$
q=\frac{\log \left(\frac{E_{j}}{E_{j+1}}\right)}{\log \left(\frac{N_{j+1}}{N_{j}}\right)},
$$

where $E_{j}$ is the norm of the error between the approximated and exact solution. $N_{j}$ denotes the number of grid points at level $j$. The same number of grid points in both coordinate directions for both fluid domains have been used.

We examine the scheme for SBP operators of order $2 s, s \in\{1,2,3\}$ in the interior. Since diagonal norms are used, the boundary accuracy is $s$ and we expect the global order of accuracy to be $s+1$ [53]. For time discretization with $s=4,100$ time steps is used, which guarantees that the time-error is small enough and that we can keep the number of time steps fixed as we refine in space. In order to make the computations faster and more efficient, a multi-block formulation in time is used [54, 55]. Here, the time interval is divided into 5 blocks.

In Tables 1-6, the convergence rates of the solution at $t=1$ for different SBP operators and different interface conditions are shown for a sequence of spatial mesh refinements. We can see from Tables 1-6 that design accuracy are obtained for both sets of interface conditions.

Next, we consider the interface conditions (25) with $N=M=39$. To show energy stability, the norm of velocities for the compressible and incompressible fluids for long time $(t=10)$ are shown in Figure 3. Clearly the norms are bounded as we expected from the theory developed in Section 4. The boundedness of the norm of the error in the solutions $U, V$ for the full system are shown in Figure 4. One can clearly see that the error is uniformly bounded, see also $[56,57,58,59]$ for similar results.

The temperature errors at $x=0$ with different SBP operators and different number of grid points are shown in Figures 5 and 6 . The error at the interface with conditions (25) and (29) for $N=M=19$ is approximately $10^{-3}$ and $10^{-2}$, respectively and for $N=M=49$ approximately $10^{-4}$ and $10^{-3}$, respectively. This shows that the error decreases at the appropriate rate as the number of grid points increases. 


\subsection{Applications}

We consider the coupled problem with either interface conditions (25) or (29) and homogeneous far-field boundary conditions derived in [52]. The background velocity fields $(\bar{u}, \bar{v})=(0.1,0),(\hat{u}, \hat{v})=(0.1,0)$ and the following initial conditions

$$
\begin{aligned}
& \rho(x, y, 0)=u(x, y, 0)=v(x, y, 0)=1, \quad(x, y) \in \Omega_{c} \\
& \tilde{p}(x, y, 0)=\tilde{u}(x, y, 0)=\tilde{v}(x, y, 0)=1, \quad(x, y) \in \Omega_{i}
\end{aligned}
$$

have been chosen. A grid consisting of $40 \times 40$ spatial nodes in each subdomain is considered. Third and fifth order accurate SBP operators in space and time, respectively, are used.

As the ratio of the specific heats, we take $\gamma=1.4$, valid for air. We have also chosen the Prandelt numbers for compressible and incompressible as $\operatorname{Pr}=0.7, \tilde{\operatorname{Pr}}=7.5$, respectively, which are typical Prandlt numbers of air and water at $17^{\circ} \mathrm{C}$. The other coefficients in (1), (5) and (28) are

$$
\epsilon=0.01, \quad \bar{\rho}=1.2 \quad \hat{\rho}=1000, \quad \bar{\mu}=1.9 \times 10^{-5} \quad \hat{\mu}=10^{-3}, \quad \alpha=1 .
$$

In the first example, we consider the interface conditions (25) and the following initial temperature

$$
\begin{aligned}
& T(x, y, 0)=0, \quad(x, y) \in \Omega_{c}, \\
& \tilde{T}(x, y, 0)=-2, \quad(x, y) \in \Omega_{s}, \quad \tilde{T}(x, y, 0)=0, \quad(x, y) \in \Omega_{i} \backslash \Omega_{s},
\end{aligned}
$$

where $\Omega_{s}$ is the blue region illustrated in the upper-left Figure 7 . By choosing the initial conditions (62), we can see how the initially colder region $\Omega_{s}$, affect the other parts of the domains, as time passes. A slightly cooler steady state compared to the initial state in $\Omega_{c}$ is obtained.

In the second example, the interface condition (29) with the following initial temperature

$$
\begin{aligned}
& T(x, y, 0)=+1, \quad(x, y) \in \Omega_{c}, \\
& \tilde{T}(x, y, 0)=-1, \quad(x, y) \in \Omega_{i},
\end{aligned}
$$

is considered. The initial temperature is illustrated in the upper-left Figure 8. The difference between initial temperature in two fluids will affect the temperature of whole domain. Also in this case the steady state in $\Omega_{c}$ is slightly cooler than the initial state. 


\begin{tabular}{lllll}
\hline \hline$N=M$ & 20 & 30 & 40 & 50 \\
\hline$\rho$ & 2.714 & 2.190 & 2.059 & 1.991 \\
$\bar{\rho} u$ & 2.234 & 2.121 & 2.180 & 2.029 \\
$\bar{\rho} v$ & 2.308 & 2.055 & 2.239 & 2.141 \\
$T$ & 2.084 & 1.944 & 2.055 & 1.983 \\
& & & & \\
$\tilde{p}$ & 2.884 & 2.836 & 2.546 & 1.990 \\
$\hat{\rho} \tilde{u}$ & 2.330 & 2.275 & 2.140 & 1.982 \\
$\hat{\rho} \tilde{v}$ & 1.677 & 2.080 & 2.208 & 2.076 \\
$\tilde{T}$ & 1.955 & 1.403 & 1.623 & 1.659 \\
\hline \hline
\end{tabular}

Table 1: Convergence rates at $t=1, \operatorname{SBP}(2,1)$ in space, $\operatorname{SBP}(8,4)$ in time and interface conditions (25).

As shown in Figures 7 and 8, the difference in initial temperature affects the temperature of both fluids and after some time $(t \approx 10$ for continuous temperature condition and $t \approx 20$ for convective condition), a uniform state is obtained.

As the third example, we focus on the acoustic waves in the compressible fluid caused by the heat sink in the incompressible domain. The interface conditions (25) with the initial temperature given in (62) and grid consisting of $60 \times 60$ spatial nodes in $\Omega_{c}$ and $\operatorname{SBP}(6,3)$, is considered.

Following [60], we plot the gradient of the density $\left(\rho_{y}\right)$ in small region near the interface for the compressible fluid to visualize the acoustic waves that arise from the temperature variation. The left plots in Figure 9 show the time development of the contour of $\rho_{y}$ for the 2 rd order $\operatorname{SBP}(2,1)$ operator and the right ones are for the 4 th order $\operatorname{SBP}(6,3)$ operator. The acoustic waves can be seen more clearly near the interface when the higher order method is used, in particular for the case $t=10$.

Next, the rate of dilatation [61] (divergence of particle velocity) at different time levels in a small region near the interface, is presented in Figure 10. As the figures illustrate, the dilatation rate turns negative as time passes, which indicates a contraction of the fluid due to the decreasing temperature. 


\begin{tabular}{lllll}
\hline \hline$N=M$ & 20 & 30 & 40 & 50 \\
\hline$\rho$ & 3.092 & 2.731 & 3.074 & 3.602 \\
$\bar{\rho} u$ & 3.244 & 2.857 & 3.027 & 3.313 \\
$\bar{\rho} v$ & 2.717 & 2.342 & 2.648 & 3.049 \\
$T$ & 2.313 & 2.308 & 2.664 & 3.018 \\
& & & & \\
$\tilde{p}$ & & & & \\
$\hat{\rho} \tilde{u}$ & 2.145 & 3.017 & 3.092 & 3.160 \\
$\hat{\rho} \tilde{v}$ & 2.687 & 2.716 & 2.838 & 3.130 \\
$\tilde{T}$ & 2.199 & 2.663 & 2.690 & 3.043 \\
\hline \hline
\end{tabular}

Table 2: Convergence rates at $t=1, \operatorname{SBP}(4,2)$ in space, $\operatorname{SBP}(8,4)$ in time and interface conditions (25).

\begin{tabular}{lllll}
\hline \hline$N=M$ & 20 & 30 & 40 & 50 \\
\hline$\rho$ & 6.389 & 3.508 & 4.494 & 4.794 \\
$\bar{\rho} u$ & 5.657 & 2.910 & 3.856 & 4.160 \\
$\bar{\rho} v$ & 6.101 & 3.047 & 3.977 & 4.271 \\
$T$ & 4.886 & 2.955 & 3.748 & 4.061 \\
& & & & \\
$\tilde{p}$ & 5.782 & 3.654 & 4.539 & 4.900 \\
$\hat{\rho} \tilde{u}$ & 5.432 & 3.108 & 3.852 & 4.160 \\
$\hat{\rho} \tilde{v}$ & 5.746 & 3.246 & 3.933 & 4.192 \\
$\tilde{T}$ & 5.515 & 3.186 & 3.915 & 4.211 \\
\hline \hline
\end{tabular}

Table 3: Convergence rates at $t=1, \operatorname{SBP}(6,3)$ in space, $\operatorname{SBP}(8,4)$ in time and interface conditions (25). 


\begin{tabular}{lllll}
\hline \hline$N=M$ & 20 & 30 & 40 & 50 \\
\hline$\rho$ & 2.011 & 2.120 & 2.159 & 2.111 \\
$\bar{\rho} u$ & 2.021 & 2.081 & 2.088 & 2.092 \\
$\bar{\rho} v$ & 1.992 & 2.055 & 2.072 & 2.100 \\
$T$ & 2.034 & 1.933 & 1.976 & 1.982 \\
& & & & \\
$\tilde{p}$ & & & & \\
$\hat{\rho} \tilde{u}$ & 1.121 & 2.144 & 2.132 & 2.112 \\
$\hat{\rho} \tilde{v}$ & 1.754 & 1.954 & 2.087 & 2.090 \\
$\tilde{T}$ & 1.921 & 1.985 & 1.999 & 2.001 \\
\hline \hline
\end{tabular}

Table 4: Convergence rates at $t=1, \operatorname{SBP}(2,1)$ in space, $\operatorname{SBP}(8,4)$ in time and interface conditions (29).

\begin{tabular}{lllll}
\hline \hline$N=M$ & 20 & 30 & 40 & 50 \\
\hline$\rho$ & 2.921 & 2.923 & 2.864 & 2.899 \\
$\bar{\rho} u$ & 3.037 & 2.999 & 2.866 & 2.897 \\
$\bar{\rho} v$ & 3.066 & 3.030 & 2.978 & 2.982 \\
$T$ & 2.864 & 2.904 & 2.862 & 2.881 \\
& & & & \\
$\tilde{p}$ & & & & \\
$\hat{\rho} \tilde{u}$ & 2.392 & 2.952 & 2.854 & 2.890 \\
$\hat{\rho} \tilde{v}$ & 3.056 & 3.994 & 2.945 & 2.961 \\
$\tilde{T}$ & 3.011 & 2.982 & 2.967 & 2.991 \\
\hline \hline
\end{tabular}

Table 5: Convergence rates at $t=1, \operatorname{SBP}(4,2)$ in space, $\operatorname{SBP}(8,4)$ in time and interface conditions (29). 


\begin{tabular}{lllll}
\hline \hline$N=M$ & 20 & 30 & 40 & 50 \\
\hline$\rho$ & 4.643 & 4.211 & 4.177 & 4.152 \\
$\bar{\rho} u$ & 5.022 & 4.321 & 4.205 & 4.179 \\
$\bar{\rho} v$ & 4.879 & 4.222 & 4.234 & 4.291 \\
$T$ & 4.896 & 4.444 & 4.234 & 4.112 \\
& & & & \\
$\tilde{p}$ & & & & \\
$\hat{\rho} \tilde{u}$ & 5.102 & 4.696 & 4.555 & 4.482 \\
$\hat{\rho} \tilde{v}$ & 4.232 & 4.242 & 4.105 & 4.079 \\
$\tilde{T}$ & 5.010 & 3.989 & 4.056 & 4.172 \\
\hline \hline
\end{tabular}

Table 6: Convergence rates at $t=1, \operatorname{SBP}(6,3)$ in space, $\operatorname{SBP}(8,4)$ in time and interface conditions (29).
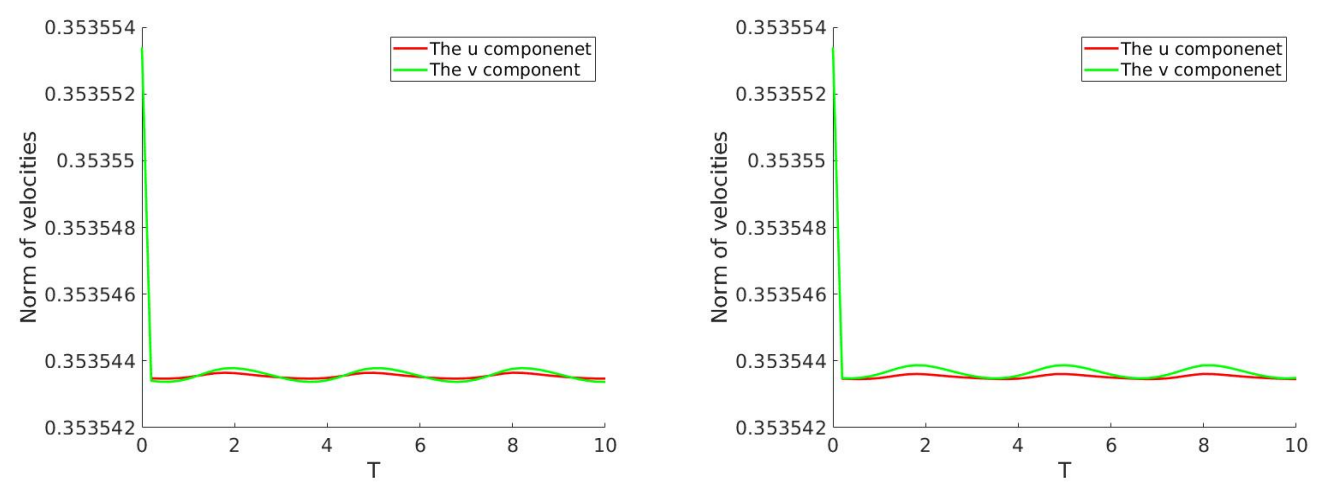

Figure 3: Norm of velocities versus time for compressible (left) and incompressible (right) fluids with interface conditions (25). 


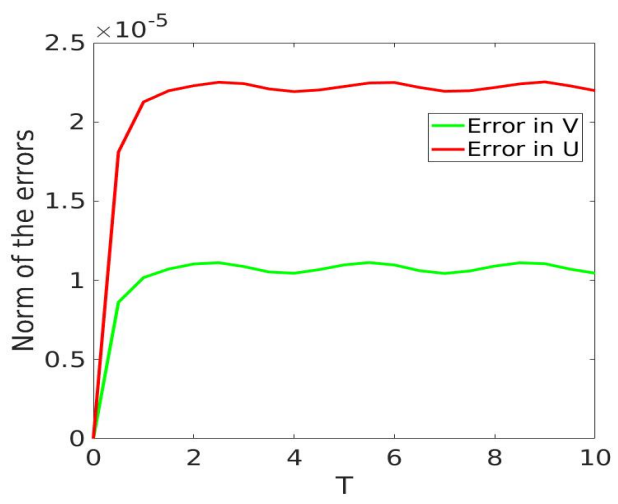

Figure 4: Norm of the errors in the solutions $U, V$ versus time for the compressible and incompressible fluids, respectively.
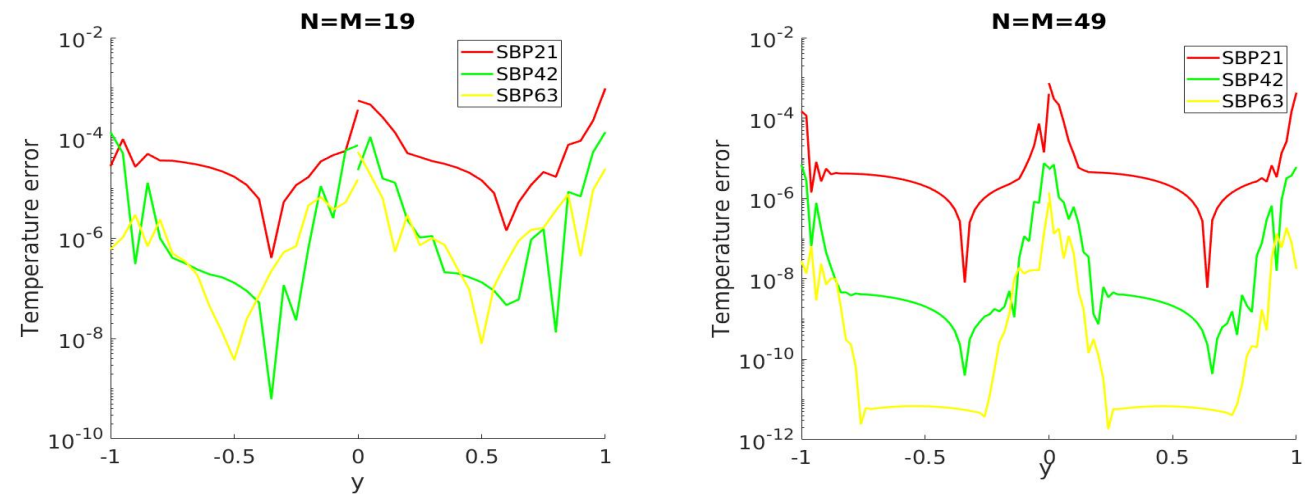

Figure 5: Semi-log plot of temperature error at $x=0$ with interface conditions (25).
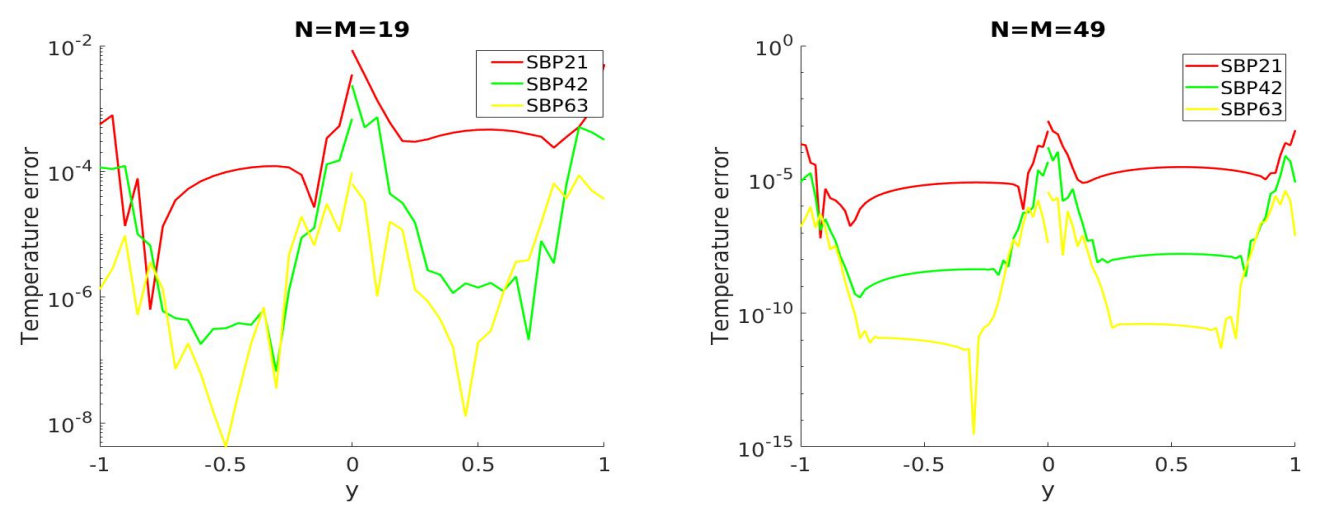

Figure 6: Semi-log plot of temperature error at $x=0$ with interface conditions (29). 


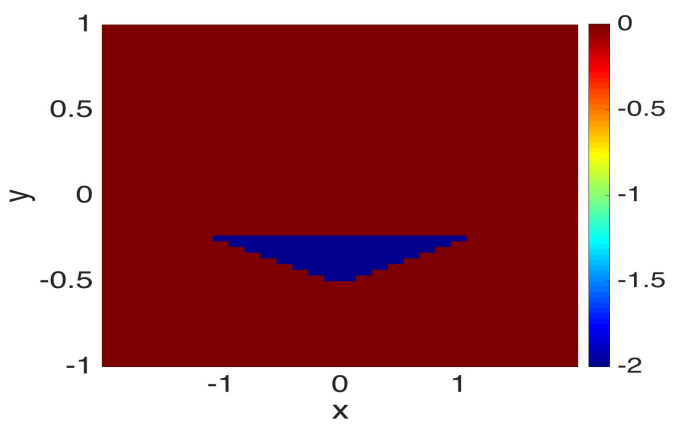

(a) $t=0$

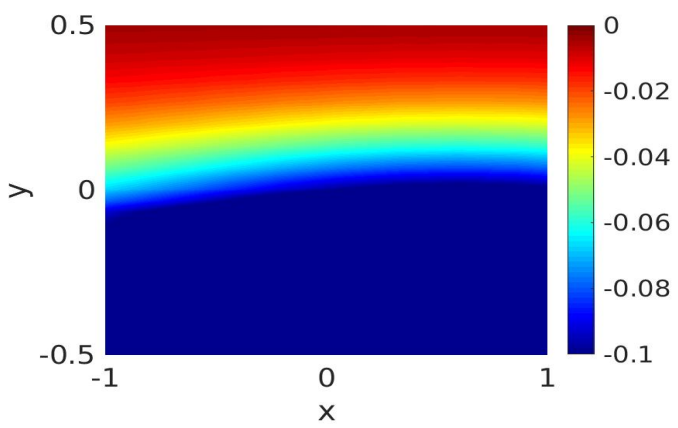

(c) $\mathrm{t}=0.5$

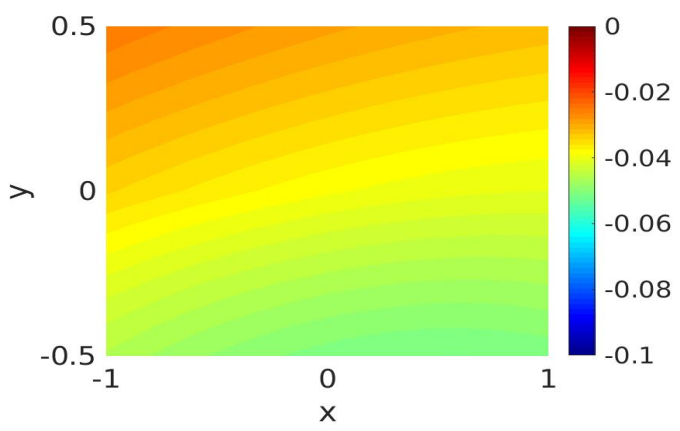

(e) $\mathrm{t}=5$

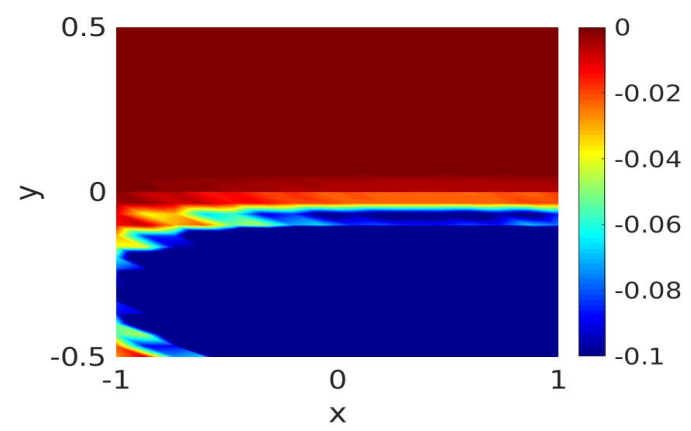

(b) $\mathrm{t}=0.05$

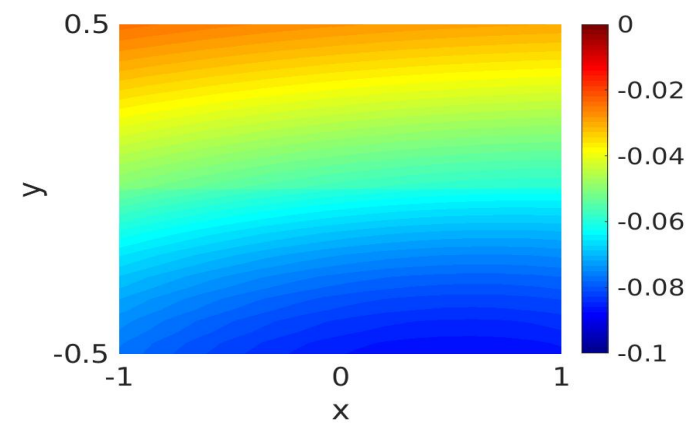

(d) $\mathrm{t}=2$

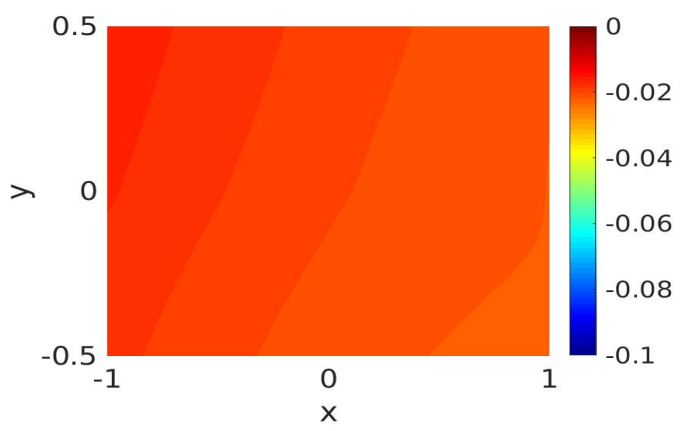

(f) $t=10$

Figure 7: A sequence (a-h) of computed temperatures with interface conditions (25) for different times using $M=N=39$ grid points. 


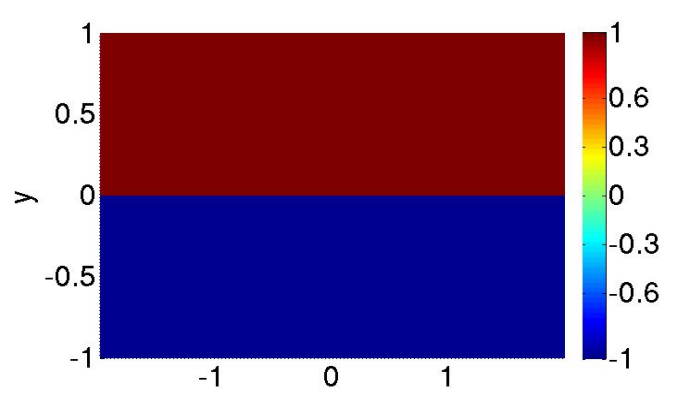

(a) $\mathrm{t}=0$

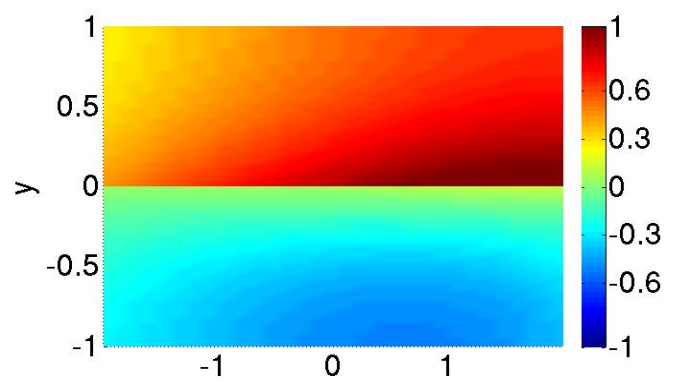

(c) $\mathrm{t}=2$

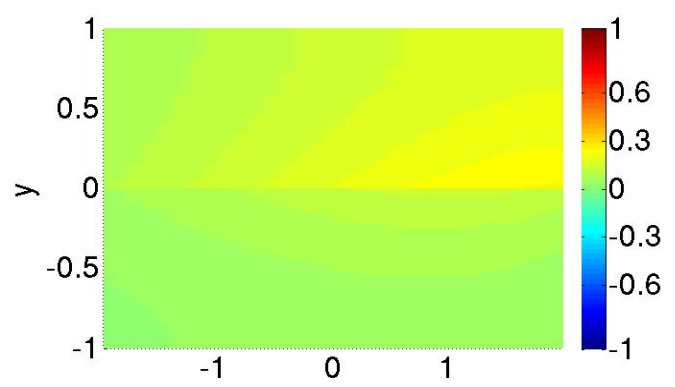

(e) $\mathrm{t}=10$

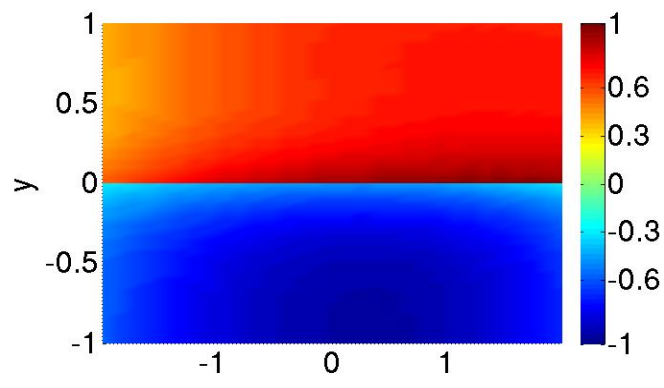

(b) $\mathrm{t}=0.5$

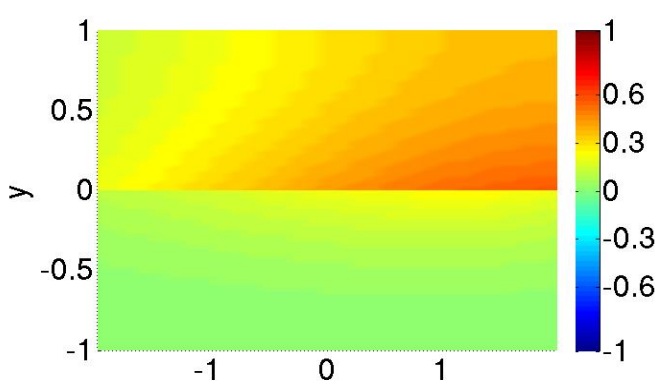

(d) $t=6$

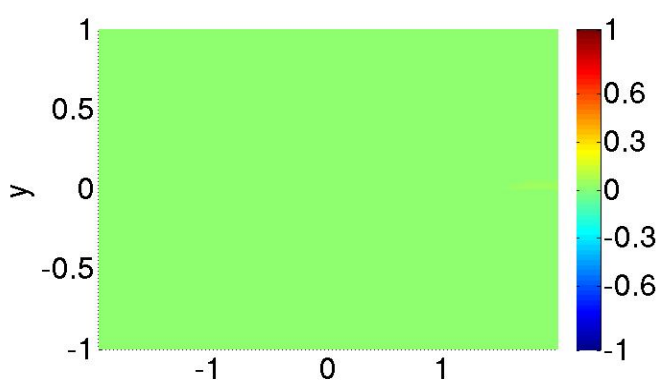

(f) $t=20$

Figure 8: A sequence (a-h) of computed temperatures with interface conditions (29) for different times using $M=N=39$ grid points. 


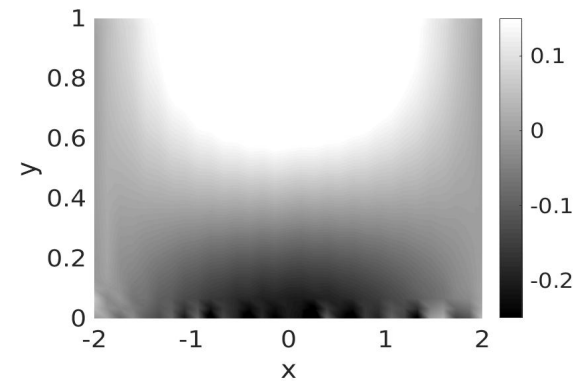

(a) $t=2$

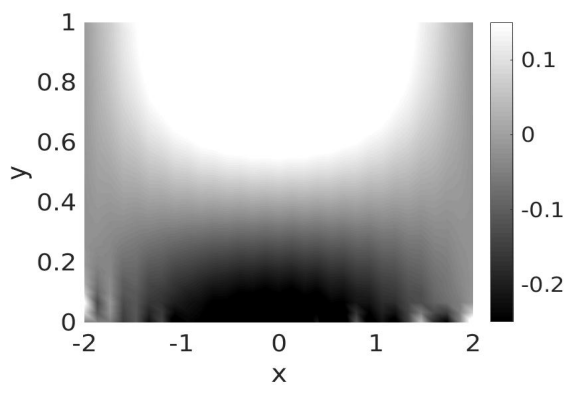

(b) $\mathrm{t}=5$

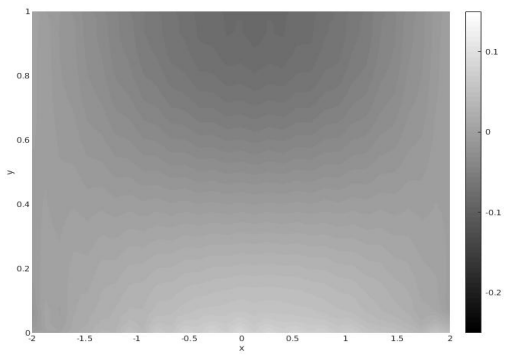

(c) $\mathrm{t}=10$

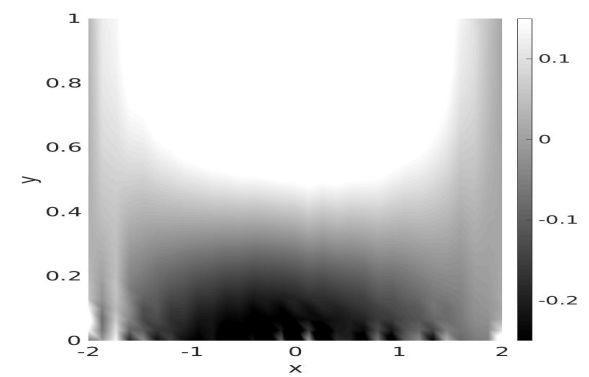

(a) $\mathrm{t}=2$

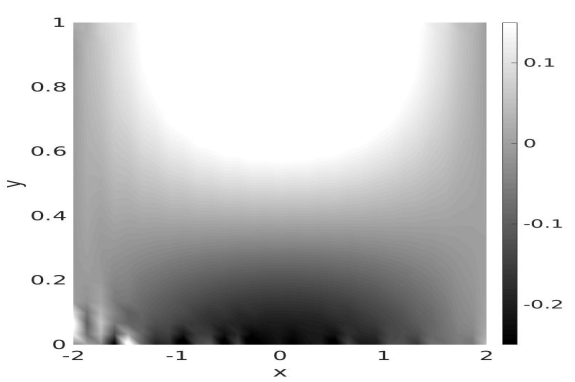

(b) $\mathrm{t}=5$

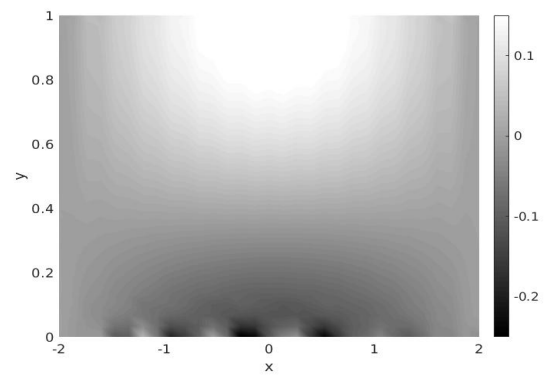

(c) $\mathrm{t}=10$

Figure 9: A sequence of contours of the density gradient, $\rho_{y}$, for the compressible fluid with interface conditions (25). (left) using $\operatorname{SBP}(2,1)$, (right) using $\operatorname{SBP}(6,3)$. 

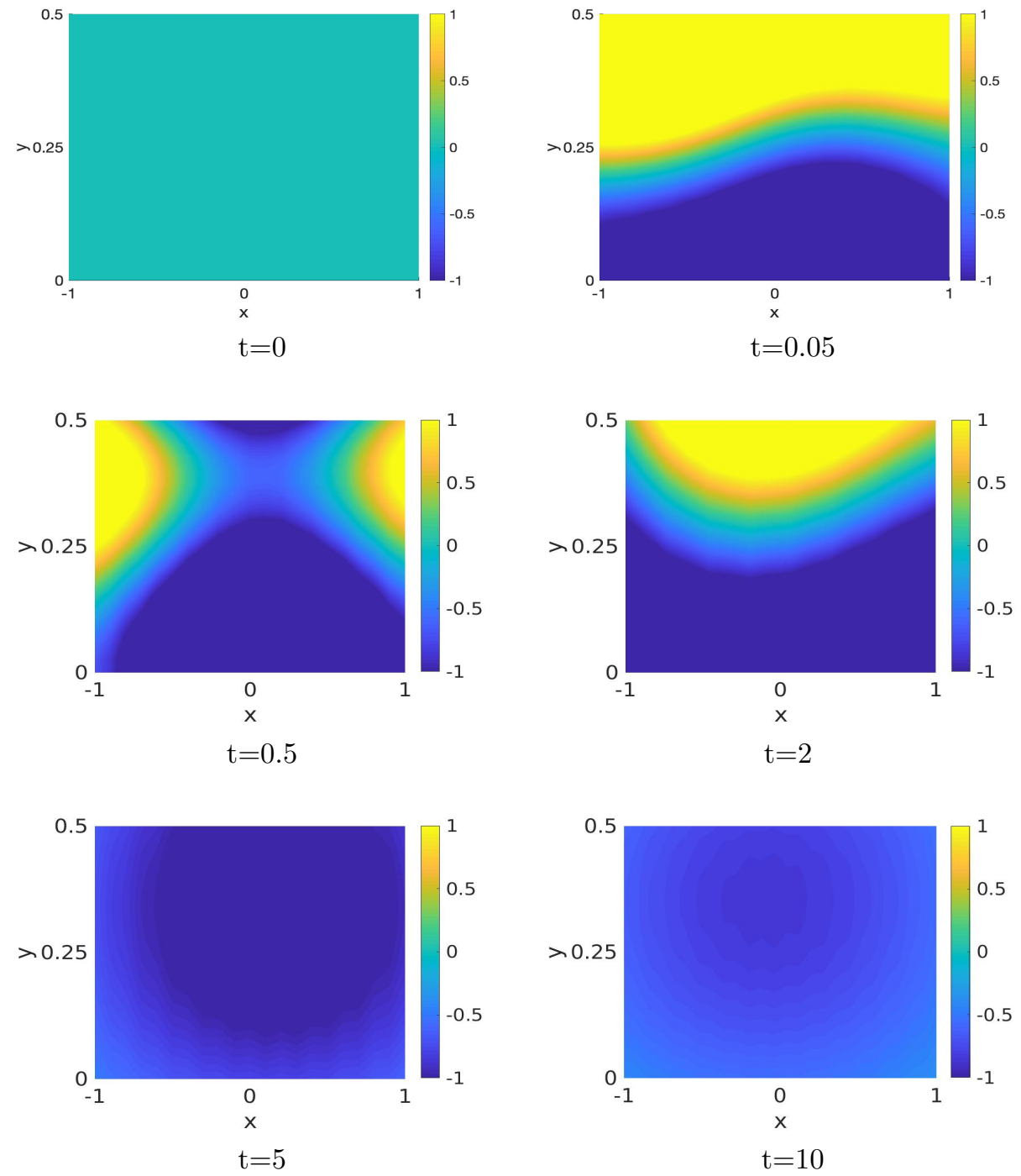

Figure 10: The rate of dilatation for the compressible fluid with interface conditions (25). 


\section{Conclusions}

We have shown that the coupling of the linearized compressible and incompressible Navier-Stokes equations at a stationary and planar interface satisfy an energy estimate when a modified norm is used. In order to obtain a sufficient number of interface conditions, the decoupled heat equation was added to the incompressible equations. Two different type of interface condition for temperature is considered. The interface conditions can be imposed strongly or weakly, which both leads to an energy bound.

The equations were discretized using finite differences on summationby-parts form with boundary and interface conditions imposed weakly. The penalty matrices which were derived in the analysis of the continuous problem lead almost automatically to stability of the discrete problem.

The convergence rates are verified by the method of manufactured solutions and the results are consistent with the theory within the SBP framework. It was also shown that the norm of both the solution and the error are bounded as time grows. The temperature errors for SBP operators of different order showed that the interface treatment is stable and accurate for all orders of accuracy.

Finally, we considered three examples where the coupled system with different initial temperatures were studied. Two types of temperature conditions at the interface were studied. In all cases, as time passes, the initial discontinuity is smeared out and a uniform slightly cooler state is obtained. It was also shown that by raising the order of the accuracy, acoustic waves indicating by density gradients that are originating from the temperature variations could be clearly seen. We also illustrated these acoustic waves using the dilatation (the divergence of the velocity) as a marker.

\section{References}

[1] J. P. Peixoto, A. H. Oort, Physics of Climate, American Institute of Physics, 1992.

[2] G. K. Vallis, Atmospheric and Oceanic Fluid Dynamics: Fundamentals and Large-scale Circulation, Cambridge University Press, 2006. 
[3] B. A. Kagan, Ocean Atmosphere Interaction and Climate Modeling, Cambridge University Press, 1995.

[4] C. Wang, S. P. Xie, J. A. Carton, Earth's Climate: The OceanAtmosphere Interaction, American Geophysical Union, 2004.

[5] S. Karni, Hybrid multifluid algorithms, SIAM Journal on Scientific Computing 17 (1996) 1019-1039.

[6] M. Sussman, P. Smereka, S. Osher, A level set approach for computing solutions to incompressible two-phase flow, Journal of Computational Physics 114 (1994) 146-159.

[7] R. Caiden, R. P. Fedkiw, C. Anderson, A numerical method for twophase flow consisting of separate compressible and incompressible regions, Journal of Computational Physics 166 (2001) 1-27.

[8] M. Boger, F. Jaegle, R. Klein, C. D. Munz, Coupling of compressible and incompressible flow regions using the multiple pressure variables approach, Mathematical Methods in the Applied Sciences 38 (2014) 458-477.

[9] F. Ghasemi, J. Nordström, Coupling requirements for multi-physics problems posed on two domains, SIAM Journal on Numerical Analysis 55 (2017) 2885-2904.

[10] J. Nordström, A roadmap to well posed and stable problems in computational physics, Journal of Scientific Computing 71 (2017) 365-385.

[11] D. C. D. R. Fernndez, J. Hicken, D. Zingg, Simultaneous approximation terms for multi-dimensional summation-by-parts operators, Journal of Scientific Computing 75 (2018) 83-110.

[12] J. Yan, J. Crean, J. Hicken, Interior penalties for summation-by-parts discretizations of linear second-order differential equations, Journal of Scientific Computing 75 (2018) 1385-1414.

[13] J. Nordström, K. Forsberg, C. Adamsson, P. Eliasson, Finite volume methods, unstructured meshes and strict stability for hyperbolic problems, Applied Numerical Mathematics 45 (2003) 453-473. 
6 CONCLUSIONS

[14] J. Nordström, S. Eriksson, P. Eliasson, Weak and strong wall boundary procedures and convergence to steady-state of the Navier-Stokes equations, Journal of Computational Physics 231 (2012) 4867-4884.

[15] H. T. Huynh, A flux reconstruction approach to high-order schemes including discontinuous Galerkin methods, 18th AIAA Computational Fluid Dynamics Conference (2007) 4079.

[16] J. Pan, H. Lin, Calculation of the sensible heat flux of the global ocean using satellite data, International Journal of Remote Sensing 33 (2012) $5289-5305$.

[17] H. Ranocha, P. Öffner, T. Sonar, Summation-by-parts operators for correction procedure via reconstruction, Journal of Computational Physics 311 (2016) 299-328.

[18] N. Wintermeyer, A. R. Winters, G. J. Gassner, D. A. Kopriva, An entropy stable nodal discontinuous Galerkin method for the two dimensional shallow water equations on unstructured curvilinear meshes with discontinuous bathymetry, Journal of Computational Physics 340 (2017) 200-242.

[19] G. J. Gassner, A skew-symmetric discontinuous Galerkin spectral element discretization and its relation to SBP-SAT finite difference methods, SIAM Journal on Scientific Computing 35 (2013) A1233-A1253.

[20] G. Tryggvason, R. Scardovelli, S. Zaleski, Direct Numerical Simulations of Gas-Liquid Multiphase Flows, Cambridge University Press, 2011.

[21] A. Caboussat, Numerical simulation of two-phase free surface flows, Archives of Computational Methods in Engineering 12 (2005) 165-224.

[22] S. Abarbanel, D. Gottlieb, Optimal time splitting for two- and threedimensional Navier-Stokes equations with mixed derivatives, Journal of Computational Physics 41 (1981) 1-43.

[23] J. Nordström, M. Svärd, Well-posed boundary conditions for the NavierStokes equations, SIAM Journal on Numerical Analysis 43 (2005) 12311255 . 
6 CONCLUSIONS

[24] B. Gustafsson, H.-O. Kreiss, J. Oliger, Time Dependent Problems and Difference Methods, Wiley, New York, 1995.

[25] H.-O. Kreiss, Initial boundary value problems for hyperbolic systems, Communications on Pure and Applied Mathematics 23 (1970) 277-298.

[26] J. Nordström, K. Mattsson, C. Swanson, Boundary conditions for a divergence free velocity-pressure formulation of the Navier-Stokes equations, Journal of Computational Physics 225 (2007) 874-890.

[27] J. Nordström, N. Nordin, D. Henningson, The fringe region technique and the fourier method used in the direct numerical simulation of spatially evolving viscous flows, SIAM Journal on Scientific Computing 20 (1999) 1365-1393.

[28] J. Nordström, C. L. Cognata, Energy stable boundary conditions for the nonlinear incompressible Navier-Stokes equations, Mathematics of Computation 88 (2018) 665-690.

[29] C. Johansson, Boundary conditions for open boundaries for the incompressible Navier-Stokes equations, Journal of Computational Physics 105 (1993) 233-251.

[30] W. D. Henshaw, A fourth-order accurate method for the incompressible Navier-Stokes equations on overlapping grids, Journal of Computational Physics 113 (1994) 13-25.

[31] M. Svärd, M. Carpenter, J. Nordström, A stable high-order finite difference scheme for the compressible Navier-Stokes equations, far-field boundary conditions, Journal of Computational Physics 225 (2007) 1020-1038.

[32] M. Svärd, J. Nordström, A stable high-order finite difference scheme for the compressible Navier-Stokes equations: no-slip wall boundary conditions, Journal of Computational Physics 227 (2008) 4805-4824.

[33] A. Gill, Atmosphere-Ocean Dynamics, Academic Press, 1982.

[34] A. Quarteroni, Numerical Models for Differential Problems, Series MS\&A, Springer, 2009. 
[35] D. Olbers, J. Willebrand, C. Eden, Ocean Dynamics, Springer Science \& Business Media, 2012.

[36] W. E. Langlois, M. O. Deville, Slow Viscous Flow, Springer Science \& Business Media, 2014.

[37] J. Nordström, J. Berg, Conjugate heat transfer for the unsteady compressible Navier-Stokes equations using a multi-block coupling, Computers \& Fluids 72 (2013) 20-29.

[38] J. R. Thome, J. Kim, Encyclopedia of Two-phase Heat Transfer and Flow II: Special Topics and Applications, World Scientific, 2016.

[39] C. Deser, M. A. Alexander, S.-P. Xie, A. S. Phillips, Sea surface temperature variability: Patterns and mechanisms, Annual Review of Marine Science 2 (2010) 115-143.

[40] D. Ferreira, C. Frankignoul, Coupled ocean-atmosphere dynamics in a simple midlatitude climate model, Journal of Climate 14 (2001) 37043723.

[41] F. Zhang, Matrix Theory Basic Results and Techniques, Springer, 1999.

[42] D. N. Arnold, F. Brezzi, B. Cockburm, L. D. Marini, Unified analysis of discontinuous Galerkin methods for elliptic problems, SIAM journal on numerical analysis 39 (2002) 1749-1779.

[43] R. A. Horn, C. R. Johnson, Topics in Matrix Analysis, Cambridge University Press, 1991.

[44] B. Strand, Summation by parts for finite difference approximations for d/dx, Journal of Computational Physics 110 (1994) 47-67.

[45] K. Mattsson, Boundary procedures for summation-by-parts operators, Journal of Scientific Computing 18 (2003) 133-153.

[46] M. Carpenter, J. Nordström, D. Gottlieb, A stable and conservative interface treatment of arbitrary spatial accuracy, Journal of Computational Physics 148 (1999) 341-365. 
6 CONCLUSIONS

[47] M. H. Carpenter, D. Gottlieb, S. Abarbanel, Time-stable boundary conditions for finite-difference schemes solving hyperbolic systems: methodology and application to high-order compact schemes, Journal of Computational Physics 111 (1994) 220-236.

[48] J. Nordström, J. Gong, E. van der Weide, M. Svärd, A stable and conservative high order multi-block method for the compressible NavierStokes equations, Journal of Computational Physics 228 (2009) 90209035 .

[49] K. Mattsson, M. H. Carpenter, Stable and accurate interpolation operators for high-order multiblock finite difference methods, SIAM Journal on Scientific Computing 32 (2010) 2298-2320.

[50] J. E. Kozdon, L. C. Wilcox, Stable coupling of nonconforming, highorder finite difference methods, SIAM Journal on Scientific Computing 38 (2016) A923-A952.

[51] L. Shunn, F. Ham, P. Moin, Verification of variable-density flow solvers using manufactured solutions., Journal of Computational Physics 231 (2012) 3801-3827.

[52] J. Berg, J. Nordström, Duality based boundary conditions and dual consistent finite difference discretizations of the Navier-Stokes and Euler equations, Journal of Computational Physics 259 (2014) 135-153.

[53] M. Svärd, J. Nordström, On the order of accuracy for difference approximations of initial-boundary value problems, Journal of Computational Physics 218 (2006) 333-352.

[54] J. Nordström, T. Lundquist, Summation-by-parts in time, Journal of Computational Physics 251 (2013) 487-499.

[55] T. Lundquist, J. Nordström, The SBP-SAT technique for initial value problems, Journal of Computational Physics 270 (2014) 86-104.

[56] J. Nordström, Error bounded schemes for time-dependent hyperbolic problems, SIAM Journal on Scientific Computing 30 (2007) 46-59. 
[57] S. Abarbanel, A. Ditkowski, B. Gustafsson, On error bounds of finite difference approximations to partial differential equations-temporal behavior and rate of convergence, Journal on Scientific Computing 15 (2000) 79-116.

[58] J. Nordström, H. Frenander, On long time error bounds for the wave equation on second order form, Journal on Scientific Computing 76 (2018) 1327-1336.

[59] D. A. Kopriva, J. Nordström, G. J. Gassner, Error boundedness of discontinuous Galerkin spectral element approximations of hyperbolic problems, Journal on Scientific Computing 72 (2017) 314-330.

[60] G. Efraimsson, J. Gong, M. Svärd, J. Nordström, An investigation of the performance of a high-order accurate Navier-Stokes code, ECCOMAS CFD Conference 2006 (2006) 1-11. 